\title{
Lactoferrin-induced myeloid-derived suppressor cell therapy attenuates pathologic inflammatory conditions in newborn mice
}

\author{
Yufeng Liu, ${ }^{1}$ Michela Perego, ${ }^{2}$ Qiang Xiao, ${ }^{3}$ Yumei He, ${ }^{3}$ Shuyu Fu, ${ }^{2}$ Juan He, ${ }^{3}$ Wangkai Liu, ${ }^{4}$ Xing Li, ${ }^{5}$ Yanlai Tang, ${ }^{4}$ Xiaoyu Li, ${ }^{4}$ \\ Weiming Yuan, ${ }^{6}$ Wei Zhou, ${ }^{6}$ Fan Wu, ${ }^{7}$ Chunhong Jia,, Qiliang Cui, ${ }^{7}$ George S. Worthen, ${ }^{8}$ Erik A. Jensen, ${ }^{8}$ Dmitry I. Cabrilovich, ${ }^{2}$ \\ and Jie Zhou' \\ 'Key Laboratory of Immune Microenvironment and Disease of the Ministry of Education, Department of Immunology, School of Basic Sciences, Tianjin Medical University, Tianjin, China. ${ }^{2}$ Wistar Institute, \\ Philadelphia, Pennsylvania, USA. ${ }^{3}$ Institute of Human Virology, Zhongshan School of Medicine, ${ }^{4}$ First Affiliated Hospital, and ${ }^{5}$ Third Affiliated Hospital, Sun Yat-sen University (SYSU), Guangzhou, China. \\ ${ }^{6}$ Guangzhou Women and Children's Medical Centre, Guangzhou, China. ${ }^{7}$ Third Affiliated Hospital, Guangzhou Medical University, Guangzhou, China. ${ }^{8}$ Children's Hospital of Philadelphia (CHOP), Philadelphia, \\ Pennsylvania, USA.
}

Inflammation plays a critical role in the development of severe neonatal morbidities. Myeloid-derived suppressor cells (MDSCs) were recently implicated in the regulation of immune responses in newborns. Here, we report that the presence of MDSCs and their functional activity in infants are closely associated with the maturity of newborns and the presence of lactoferrin (LF) in serum. Low amounts of MDSCs at birth predicted the development of severe pathology in preterm infants - necrotizing enterocolitis (NEC). In vitro treatment of newborn neutrophils and monocytes with LF converted these cells to MDSCs via the LRP2 receptor and activation of the NF-KB transcription factor. Decrease in the expression of LRP2 was responsible for the loss of sensitivity of adult myeloid cells to LF. LF-induced MDSCs (LF-MDSCs) were effective in the treatment of newborn mice with NEC, acting by blocking inflammation, resulting in increased survival. LF-MDSCs were more effective than treatment with LF protein alone. In addition to affecting NEC, LF-MDSCs demonstrated potent ability to control ovalbumin-induced (OVA-induced) lung inflammation, dextran sulfate sodium-induced (DSS-induced) colitis, and concanavalin A-induced (ConAinduced) hepatitis. These results suggest that cell therapy with LF-MDSCs may provide potent therapeutic benefits in infants with various pathological conditions associated with dysregulated inflammation.

\section{Introduction}

Early life, especially the first weeks after birth, is characterized by high vulnerability to infection and inflammation-mediated tissue injury. Dysregulation of inflammatory responses and hostmicrobial interactions play a central role in the development of the most common and severe neonatal morbidities: bronchopulmonary dysplasia, necrotizing enterocolitis (NEC), and sepsis. Together, these conditions contribute to over half of all deaths in extremely preterm infants and result in health care costs totaling over $\$ 4$ billion annually in the US (1-4). NEC is the most common gastrointestinal emergency in preterm infants. The disorder is characterized by ischemic necrosis of the intestinal mucosa, severe bowel and systemic inflammation, invasion of enteric gas-forming organisms into the surrounding organs and systemic bloodstream, and dissection of gas into the bowel wall and por-

Related Commentary: p. 4080

Authorship note: DIC and JZ are co-senior authors.

Conflict of interest: The authors have declared that no conflict of interest exists. Copyright: () 2019, American Society for Clinical Investigation.

Submitted: February 14, 2019; Accepted: July 9, 2019; Published: September 4, 2019

Reference information: J Clin Invest. 2019;129(10):4261-4275.

https://doi.org/10.1172/JCl128164. tal venous system $(5,6)$. NEC develops in $7 \%-11 \%$ of infants born with birth weights of less than $1,500 \mathrm{~g}$ (7-9). The estimated mortality rate due to NEC is nearly $30 \%$ and approaches $100 \%$ among those with the most severe form, NEC totalis (10). The systemic inflammatory response that accompanies the acute intestinal injury in NEC adversely affects multiple organs, placing survivors at high risk for chronic deficits in lung and renal function and lifelong neurologic impairment (11-13).

Microbial pathogens are recognized by the innate immune system via diverse pattern recognition receptors that trigger proinflammatory and antimicrobial responses. However, the same response is also triggered by commensal organisms that colonize mucosal surfaces at birth and therefore must be regulated to avoid harmful inflammation. Among the factors involved in the regulation of immune responses in newborns (14), the prominent role of myeloid-derived suppressor cells (MDSCs) became apparent in recent years. MDSCs have emerged as major regulators of inflammation and immune function in many pathological conditions. MDSCs consist of 2 groups of cells: polymorphonuclear (PMN-MDSCs) and monocytic (M-MDSCs) (15). These cells share characteristics with neutrophils (PMN) and monocytes (MON), but comparatively, display a relatively immature phenotype and morphology, increased levels of ROS and NO production, and higher expression of arginase (ARG1), prostaglandin $\mathrm{E}_{2}$ (PGE2), and a 

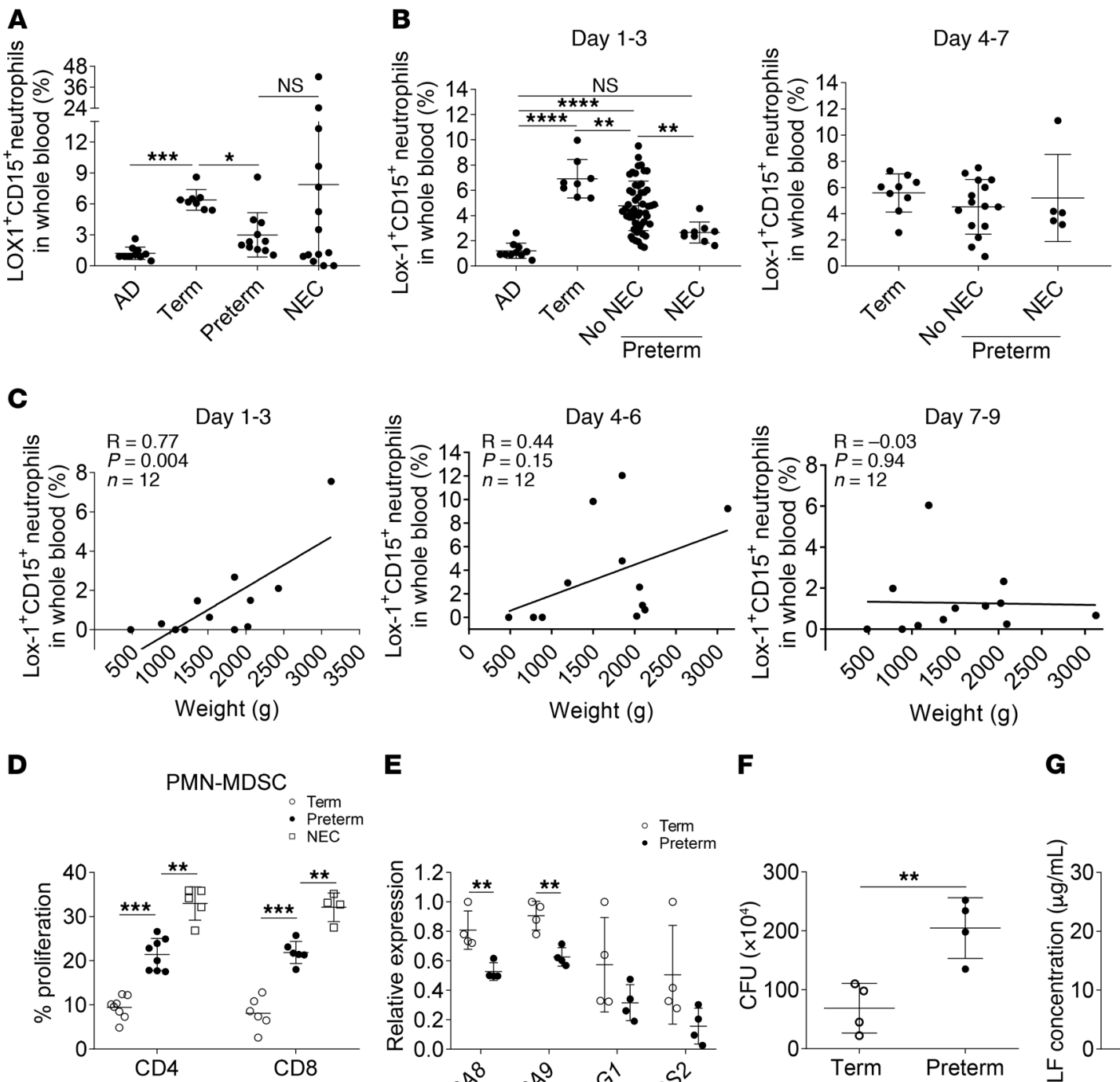

$\mathbf{E}$

$\mathbf{F}$

G
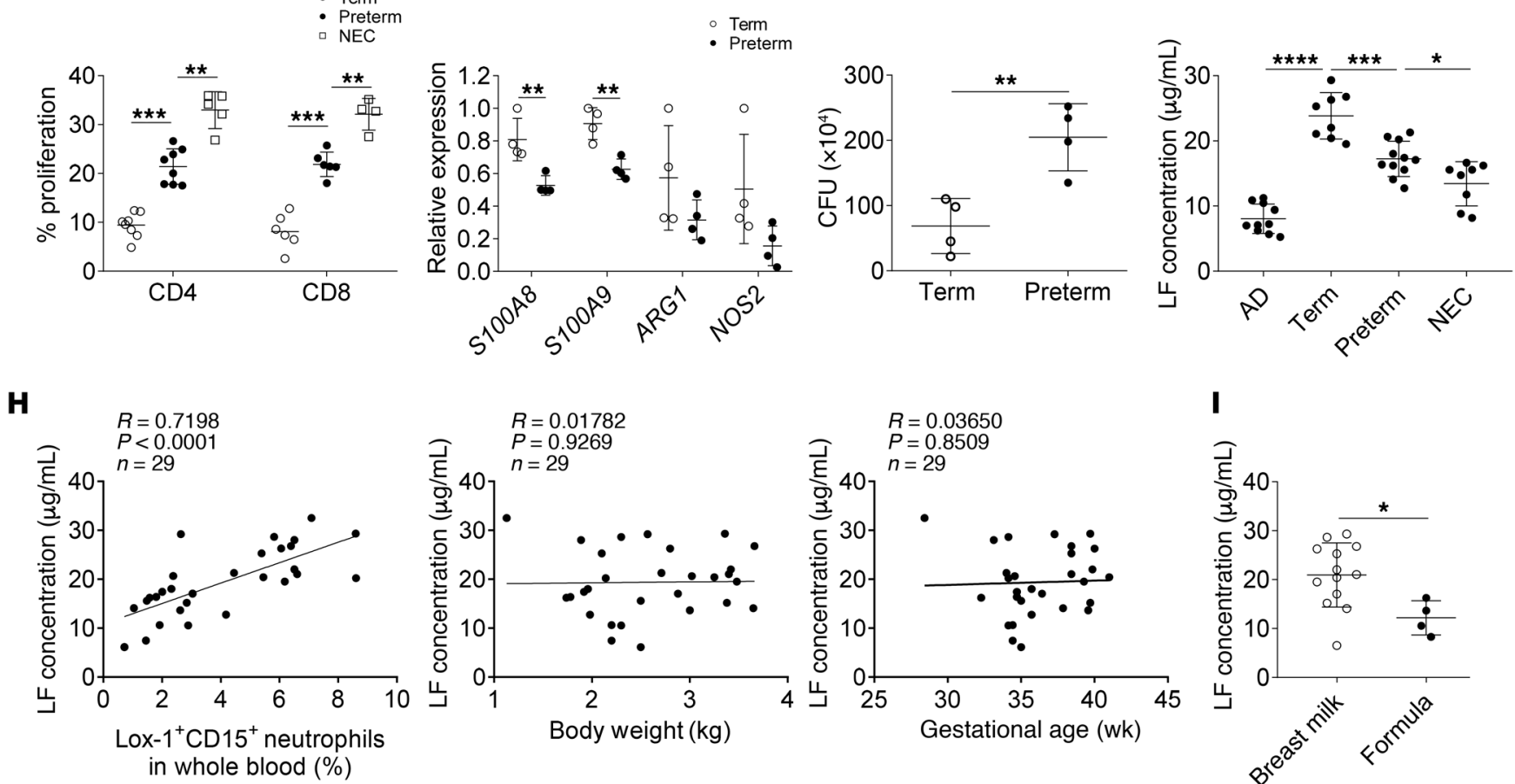

Figure 1. Clinical significance of MDSCs in infants. (A) Percentages of Lox1+CD15 PMN-MDSCs in adults (AD) ( $n=10)$, full-term (term) ( $n=8)$, and preterm $(n=11)$ infants and patients with NEC $(n=14)$. Samples from NEC patients were collected at diagnosis ( 6 to 66 days after birth). In all other cases, samples were collected between days 1 and 4 after birth. (B) Percentages of PMN-MDSCs in infants who later developed or did not develop NEC. Samples were collected on days 1-3 (AD, $n=10$; term, $n=8$; preterm, no NEC, $n=51$; preterm with NEC, $n=9$ ) or 4-7 (term, $n=9$; preterm, no NEC, $n=15$; preterm with NEC, $n=5$ ) after birth. (C) Correlation between proportion of PMN-MDSCs and body weight of infants at different age. (D) Functional activity of PMN-MDSCs from infants using T cells stimulated with CD3/CD28 antibodies. T cell proliferation was measured in triplicate with CFSE labeling $(n=4-8)$. (E) Expression of indicated genes in PMN-MDSCs measured by quantitative reverse-transcriptase PCR (qRT-PCR) $(n=4)$. (F) Antibacterial activity of PMN-MDSCs against $E$. coli $(n=4)$. (C) Concentrations of LF in plasma from adults and newborns determined by ELISA ( $n=8-11)$. (H) Correlation between LF concentration (plasma) and proportion of PMN-MDSCs, body weight, or gestational age of newborns. (I) LF concentration in plasma of infants who were fed with breast milk $(n=13)$ or formula $(n=$ 4). In all panels, individual results and mean \pm SD are shown. $P$ values were calculated using 2-sided Student's $t$ tests (E, F, and I) or 1-way ANOVA followed by Tukey-Kramer multiple-comparisons test (A, B, D, and G). ${ }^{*} P<0.05 ;{ }^{* *} P<0.01$; ${ }^{* *} P<0.001$; ${ }^{* * *} P<0.0001$. For C and $\mathbf{H}$, Spearman's correlation coefficient was calculated, and actual $P$ values are shown. 
number of antiinflammatory cytokines, including IL-10 (16-18). A primary feature of MDSCs is their potent ability to inhibit immune responses, including those of T cells, B cells, and NK cells (19).

Until recently, MDSCs were exclusively associated with various pathologic conditions and pregnancy and were not known to be present in steady-state conditions in healthy individuals (19). Recent studies challenged this paradigm by demonstrating expansion of MDSCs in healthy neonates $(20,21)$. Similarly to MDSCs observed in chronic inflammation and cancer, these cells suppressed $\mathrm{T}$ cells in a contact-dependent manner and reduced IFN- $\gamma$ production $(20,22,23)$. PMN-MDSCs from cord blood (CB) directly inhibited Th1 response and induced Th2 response and Tregs (24). However, in contrast to MDSCs in cancer, MDSCs in newborn mice have enriched gene expression associated with greater antimicrobial activity and more potent ability to kill pathogens than PMNs or MONs obtained from adult mice (21). Neonatal PMN-MDSCs show reduced apoptosis and immunosuppressive activity upon infection with E. coli (25). S100A8/A9 proteins (one of the hallmarks of MDSCs) prevented death from septic shock in newborn mice (26). Evidence from humans showed that S100A9 secretion protected neonates from sepsis by regulating MyD88-dependent gene programs (27). In a recent study, we demonstrated that MDSCs could be an important component of natural host defense mechanisms in newborns (21). However, very preterm infants, not those born at or near term gestation, are at the greatest risk of infection and inflammatory injury. The clinical significance of MDSCs in preterm infants remains unclear. Our previous study demonstrated the important role of lactoferrin (LF) in the regulation of MDSCs in newborns (21). It opened an opportunity to leverage LF for the development of cell-based therapy of inflammatory and autoimmune conditions. Here, we report that the presence of MDSCs and their functional activity in infants are closely associated with the maturity of newborns and predict the development of NEC in preterm infants. We found that, in mice and humans, LF was able to convert myeloid cells to potent MDSCs only in infants, but not in adults. Moreover, we identified the mechanism of this phenomenon. Finally, we demonstrated the ability of cell therapy with LF-induced MDSCs (LF-MDSCs) to control different pathological inflammatory conditions, including NEC, lung and colon inflammation, and hepatitis.

\section{Results}

MDSCs in preterm infants. Analysis of peripheral blood (PB) was performed in parallel at 2 institutions, SYSU and CHOP, using the same phenotypic criteria and protocols. PMN-MDSCs in whole blood were evaluated as LOX $-1^{+} \mathrm{CD} 15^{+}$cells (28) and in PBMCs

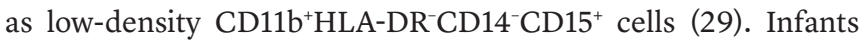
that were born at full term (gestational age $\geq 37$ weeks) had much higher proportions of $\mathrm{LOX}-1^{+} \mathrm{CD} 15^{+} \mathrm{PMN}-\mathrm{MDSC}$ in the $\mathrm{PB}$ than healthy adult volunteers. The amount of detectable PMN-MDSCs was significantly lower $(P=0.0007)$ in preterm infants (gestational age $<37$ weeks) (Figure $1 \mathrm{~A}$ ). Similar results were obtained when PMN-MDSCs were evaluated as low-density CD11b ${ }^{+}$HLA$\mathrm{DR}^{-} \mathrm{CD} 14^{-} \mathrm{CD}^{+} 5^{+}$cells (Supplemental Figure 1A; supplemental material available online with this article; https://doi.org/10.1172/ JCI128164DS1). In contrast, no differences were observed in the total number of PMNs (Supplemental Figure 1B).
Infants with NEC showed variable presence of PMN-MDSCs at the time of diagnosis (mostly between days 6 and 24 after birth) (Figure 1A). To assess the possible link between PMN-MDSCs and the development of NEC, we evaluated populations of PMNMDSCs in preterm infants before the development of NEC. On days 1-3 after birth, preterm infants who eventually developed NEC had significantly $(P=0.002)$ lower numbers of PMN-MDSCs than preterm infants who did not develop NEC. These differences were not observed on days 4-7 after birth (Figure 1B). No differences in the total presence of PMN were observed between the groups (Supplemental Figure 1C). To exclude the possible influence of weight and gestational age on the association between PMN-MDSC level and the development of NEC (risk of NEC is inversely proportional to birth weight and gestational age), we evaluated samples, collected 1-3 days after birth, from infants with similar birth weights (1,700-2,000 g) and gestational ages (31-34 weeks). The proportion of PMN-MDSCs measured during the first days after birth in newborns that later developed NEC were significantly lower $(P<0.05)$ than in infants who did not develop NEC (Supplemental Figure 1D). On days 1-3, there was a strong positive correlation $(P=0.004)$ between the birth weight of the infant and the presence of PMN-MDSCs. This correlation gradually disappeared with time (Figure 1C). No correlation with the total population of PMNs was observed (Supplemental Figure 1E).

PMN-MDSCs from healthy full-term infants had potent T cellsuppressive activity. This activity was substantially lower in preterm infants and even lower in patients with NEC (Figure 1D). Decreased ability of PMN-MDSCs to inhibit T cells in preterm infants was associated with lower expression of factors responsible for suppressive activity of MDSCs (21): S100A9, S100A8, ARG1, and NOS2 (Figure 1E). PMN-MDSCs from preterm infants had lower antibacterial activity than cells from full-term infants (Figure 1F).

We tested for the presence of M-MDSCs in PBMCs of infants based on a standard set of criteria CD11b ${ }^{+}$HLA-DR ${ }^{-}$CD $14^{+} \mathrm{CD} 15^{-}$ cells (29). As was the case for PMN-MDSCs, the presence of M-MDSCs in infants was dramatically higher than in adults. In preterm infants, it was significantly lower $(P=0.0007)$ than in infants born full term (Supplemental Figure 2A). The ability of M-MDSCs to suppress $\mathrm{T}$ cell proliferation was substantially reduced in preterm infants and patients with NEC as compared with healthy full-term infants (Supplemental Figure 2B).

LF may be involved in the accumulation of MDSCs in newborn mice (21), but its significance in human infants remained unknown. We investigated the link between LF and PMNMDSCs in infants. The amount of LF in the serum of preterm infants was significantly lower $(P=0.0002)$ than in full-term infants, and patients with NEC had less LF than preterm infants (Figure 1G). The presence of LF correlated closely with the level of PMN-MDSCs in infants $(R=0.71, P<0.0001)$, but not with birth weight or gestational age (Figure $1 \mathrm{H}$ ). The amount of LF in the serum of infants fed with formula (which contains considerably less LF than human milk) was dramatically lower than in infants who received breast milk (Figure 1I). These results indicate that both the presence and the activity of MDSCs were closely associated with the maturity of the infants and the level of LF in serum.

LF converts PMNs and MONs to MDSCs in newborns, but not in adults. We asked whether LF exposure alone could generate 
A
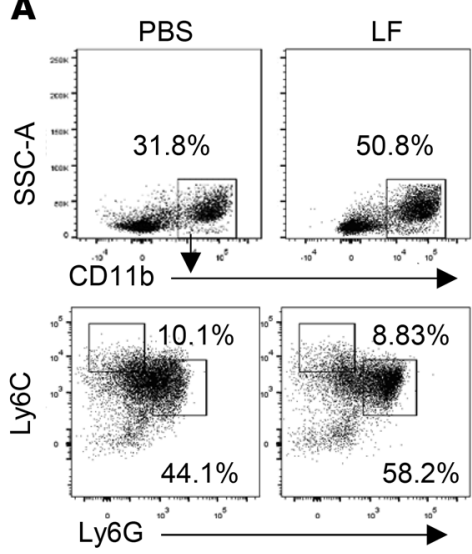

C
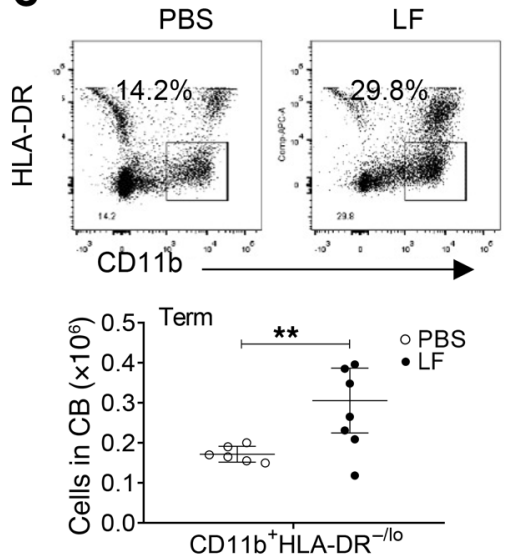
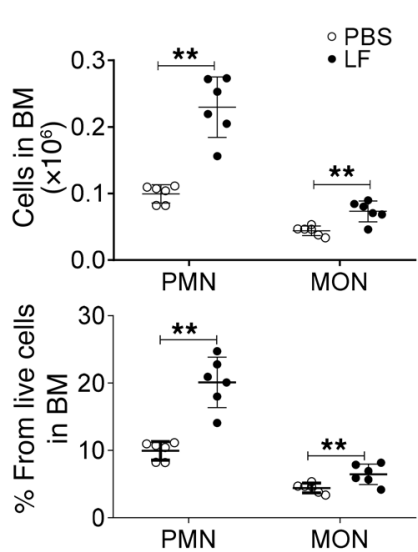

B
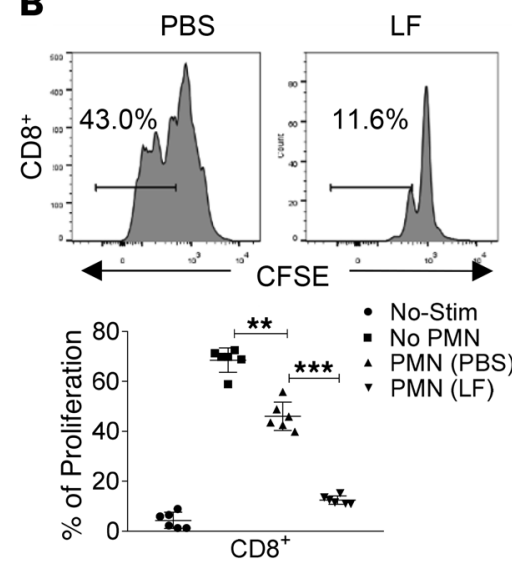

D PBS

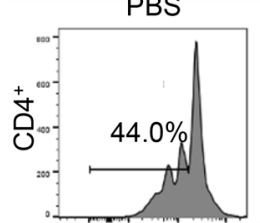

$\mathrm{LF}$
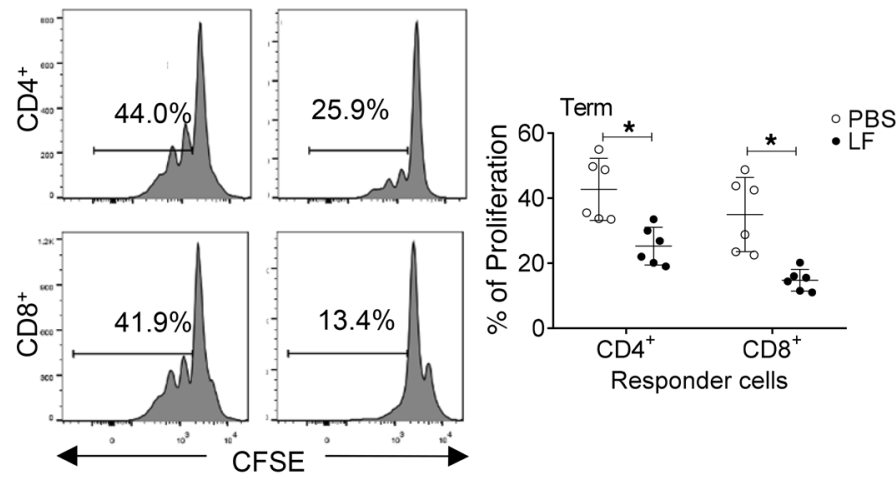

E

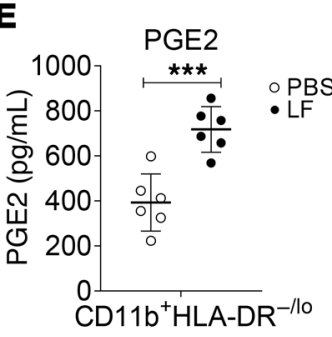

$\mathbf{F}$
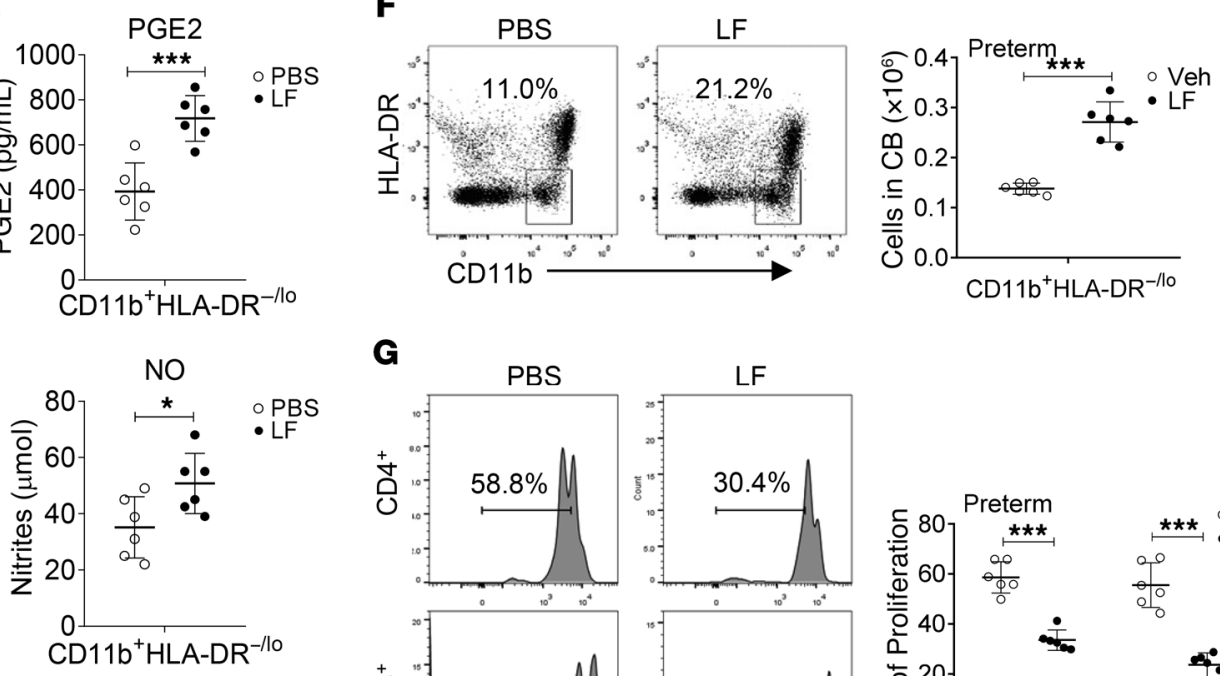

LF
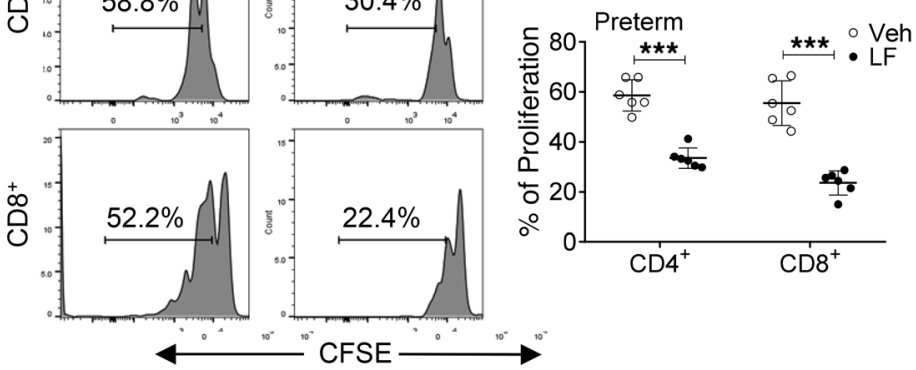
Figure 2. LF converts PMN and MON to MDSCs. (A) BM cells from 2-week-old mice were treated with LF or vehicle (PBS) in the presence of GM-CSF for 2 days. Typical example of flow cytometry (left), absolute numbers, and proportion of PMN and MON (right) $(n=6)$. (B) Suppressive activity of LF-induced PMN-MDSCs against proliferation of OT-I-derived $\mathrm{CD}^{+} \mathrm{T}$ cells stimulated with cognate peptide $(n=6)$. Nonstimulated T cells (No-stim) and stimulated T cells without PMN (No-PMN) were used as controls. (C) Absolute numbers of CD11b+HLA-DR-1/0 cells in PBS- or LF-treated CB mononuclear cells for 2 days $(n=6)$. (D) Suppression of LF-induced CD11b+HLA-DR-/lo cells from CB mononuclear cells against $T$ cells stimulated with $C D 3 / C D 28$ antibodies. T cell proliferation was measured in triplicate by CFSE dilution $(n=6)$. (E) PGE2 and NO in myeloid cells from PBS- or LF-treated CB mononuclear cells $(n=6)$. (F) Absolute numbers of myeloid cells in PBS- or LF-treated preterm infant-derived CB mononuclear cells for 2 days $(n=6)$. (C) CD11b+HLA-DR-/lo were sorted from PBS- or LF-treated preterm infant CB mononuclear cells, followed by incubation with $T$ cells stimulated with CD3/CD28 antibodies. T cell proliferation was measured in triplicate by CFSE labeling $(n=6)$. In all plots, the results of individual experiments and mean $\pm S D$ are shown. $P$ values were calculated using 2 -sided Student's $t$ tests (A and C-G) or 1-way ANOVA followed by Tukey-Kramer multiple-comparisons test (B). ${ }^{*} P<0.05 ;{ }^{* *} P<0.01$; ${ }^{* * *} P<0.001$.

MDSCs. We used BM cells from mice of different ages: 2 weeks old and 6-8 weeks old. A previous study demonstrated that 2 weeks was the time when MDSC activity was lost, and adult 6- to 8-week-old mice don't have detectable MDSC activity (21). BM cells were cultured for 48 hours with GM-CSF and LF. In the BM cells of 2-week-old mice, treatment with LF caused substantial expansion of both PMN and MON (Figure 2A). The effect was observed at a LF concentration of greater than $300 \mu \mathrm{g} / \mathrm{ml}$ (Supplemental Figure 3A). We found that treatment with LF did not affect $\mathrm{T}$ cell proliferation in response to stimulation with CD3/ CD28 antibodies (Supplemental Figure 3B) and that the production of IFN- $\gamma$ was moderately suppressed (Supplemental Figure 3C). LF converted PMN to highly immune-suppressive PMN-MDSCs (Figure 2B). Although the population of MON was relatively small, LF also enhanced suppressive activity of these cells (Supplemental Figure 3D). Twenty-four-hour treatment caused only a partial effect, and treatment lasting longer than 48 hours did not substantially increase the effect. Similar experiments were performed with mononuclear cells isolated from human CB. Twoday treatment with LF expanded the population of myeloid cells (Figure 2C and Supplemental Figure 3E) and converted them into cells with potent ability to suppress $\mathrm{CD} 4^{+}$and $\mathrm{CD} 8^{+} \mathrm{T}$ cells (Figure 2D). We next tested to determine whether LF could affect PMNs isolated from human CB. Two-day treatment of CB PMN with LF, but not with PBS, resulted in the acquisition of potent suppressive activity (Supplemental Figure 3F). When similar experiments were performed with BM of 6- to 8-week-old mice or PBMCs isolated from healthy adult donors, no effect of LF on suppressive activity of myeloid cells was observed (Supplemental Figure 4, A-D).

A previous study demonstrated that the suppressive activity of PMN-MDSCs in newborns was mediated via S100A8/A9 proteins and increased production of PGE2, whereas M-MDSCs were mediated via increased expression of Nos 2 and production of NO (21). We found that treatment of newborn mouse BM cells with LF caused upregulation of the expression of S1OOa9 and S1OOa8 (Supplemental Figure 5A) and PGE2 production (Supplemental Figure
5B) in PMN-MDSCs and upregulation of S100a9, S100a8, and Nos2 (Supplemental Figure 5C) as well as increased NO production by M-MDSCs (Supplemental Figure 5D).

Similar results were obtained with myeloid cells $\left(\mathrm{CD} 11 \mathrm{~b}^{+}\right.$ $\mathrm{HLA}^{-D^{-/ 10}}$ ) from CB obtained from full-term infants (Figure $2 \mathrm{E}$ and Supplemental Figure 5E). However, preterm infants more commonly develop inflammatory-mediated pathological conditions. We asked whether LF could cause expansion of myeloid cells and their conversion to MDSCs. Treatment of CB mononuclear cells from preterm infants caused expansion of suppressive M-MDSCs (Figure 2, F and G).

Potent antibacterial activity is a prominent feature of newborn MDSCs (21). We assessed the effect of LF on this activity of myeloid cells. BM cells from 2-week-old mice treated with LF for 48 hours demonstrated increased killing of E. coli and Candida albicans (Figure 3, A and B). Similar results were obtained with Cronobacter sakazakii, which can result in fatal infectious disease in infants (Figure 3C). Treatment with LF was associated with a substantial increase in phagocytic activity by MDSCs (Figure 3D). Similar experiments were performed with human myeloid cells from CB. LF induced substantial increases in both phagocytic activity (Figure 3E) and killing of pathogens (Figure 3F).

Mechanism of LF-MDSC expansion in newborns. Activation of $\mathrm{NF}-\kappa \mathrm{B}$ transcription factor is one of the major downstream targets of $\operatorname{LF}(30,31)$. As expected, treatment of BM cells from 2-week-old mice with LF caused accumulation of p65 (RelA) in the nucleus, which was cancelled by the selective NF- $\kappa$ B inhibitor JSH23 (Figure $4 \mathrm{~A}$ ). Inhibition of NF- $\mathrm{BB}$ abrogated LF-induced accumulation (Figure 4B) and suppressive activity of PMN-MDSCs (Figure 4C) and M-MDSCs (Supplemental Figure 6A). It was associated with inhibition of major mechanisms of LF-mediated MDSC activation: production of S100A9 (Figure 4D), PGE2 (Figure 4E), and NO (Supplemental Figure 6B). Similar experiments were performed with myeloid cells from human CB. JSH23 cancelled LF-induced accumulation (Figure $4 \mathrm{~F}$ ), suppressive activity (Figure $4 \mathrm{G}$ ) of myeloid cells, and upregulation of S100A9, PGE2, and NO (Supplemental Figure 6, C-E). We further evaluated the expression of phosphorylated NF- $\kappa \mathrm{B}$ (p65) in myeloid cells isolated from infants. Preterm infants had significantly lower $(P=0.02)$ phosphorylated p65 levels than children born at term (Supplemental Figure 6F). Using siRNA, we silenced p65 in myeloid cells (Supplemental Figure 6G) and found that it abrogated LF-induced expansion of PMN- and M-MDSCs (Supplemental Figure 6H) and acquisition of suppressive activity by these cells (Supplemental Figure 6, I and $\mathrm{J})$. All these results suggest that NF- $\mathrm{BB}$ may mediate acquisition of MDSC characteristics by LF in myeloid cells.

Our results indicate that myeloid cells in mouse and human newborns were sensitive to LF, whereas myeloid cells from adults were not. This raised the question, why does LF affect myeloid cells only in newborns? To address this question, we evaluated the expression of different receptors capable of binding LF: low density lipoprotein receptor-related protein 1 (LRP1), LRP2, intelectin 1 (ITLN1), and ITLN2. Myeloid cells in newborn mice demonstrated much stronger expression of $\operatorname{Lrp} 2$ than It $\ln 1$, It $\ln b$, or Lrp1 (Figure 5A). There was a drop in the expression of Lrp2 with age (Figure $5 \mathrm{~B}$ ), which translated to a substantial reduction of Lrp2 expression on the cell surface (Figure 5C). Similar expression 
A

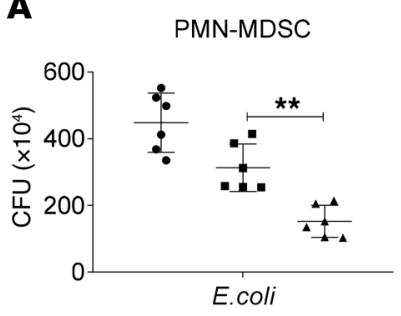

C
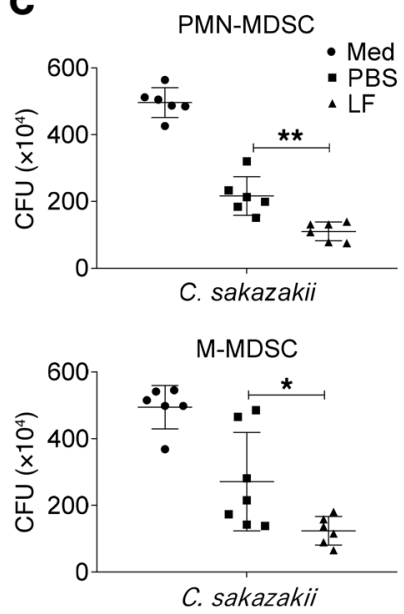

$\mathbf{E}$

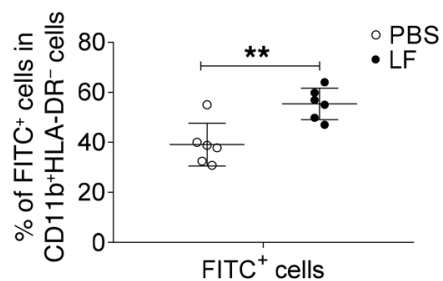

M-MDSC

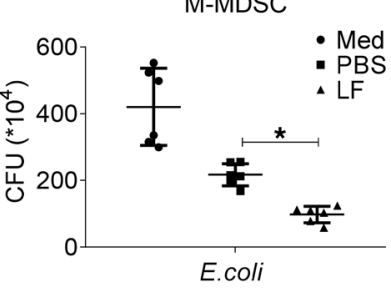

D

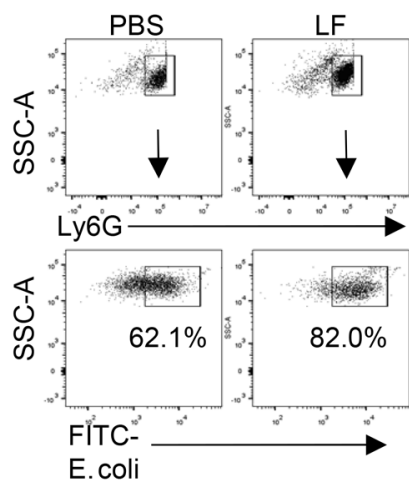

$\mathbf{F}$

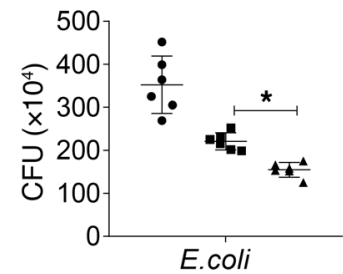

B
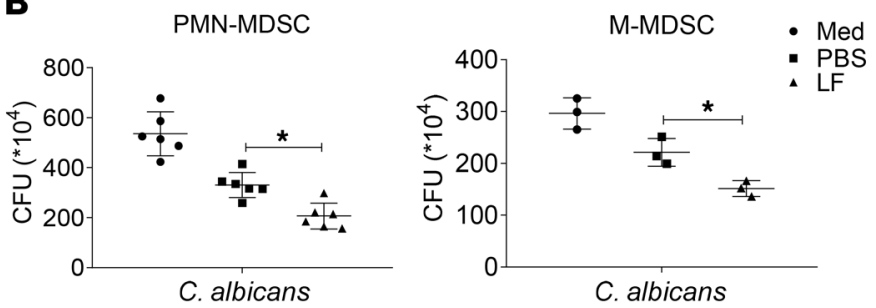

PMN-MDSC
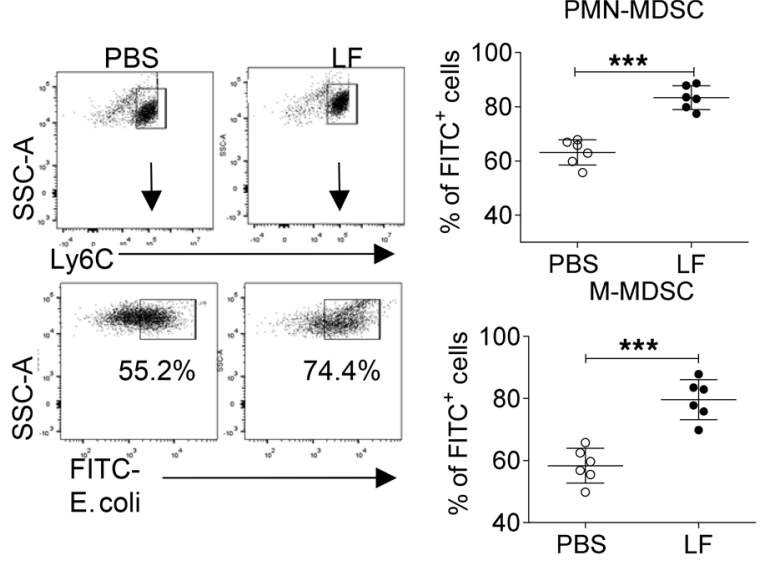

Figure 3. Antibacterial activity of LF-MDSCs. (A and B) Antibacterial activity of PMN-MDSCs or M-MDSCs isolated from PBS- or LF-treated newborn BM cells against $E$. coli $(\mathbf{A}, n=6)$ or with $C$. albicans (B, $n=3-6)$. CFU were calculated after 24 hours of incubation. Medium (Med) alone was used as control. (C) Antibacterial activity of PMN-MDSCs and M-MDSCs against C. sakazakii $(n=6)$. (D) Phagocytosis of FITC-labeled pHrodo E. coli BioParticles (100 $\mu$ g/ $1 \times 10^{5}$ cells for 90 minutes) by LF-induced mouse PMN-MDSCs or M-MDSCs. Typical example (left) and cumulative results (right) are shown ( $n=6)$. (E) Phagocytosis of FITC-labeled pHrodo E. coli BioParticles by LF-induced human MDSCs from CB $(n=6)$. (F) Antibacterial activity of myeloid cells from PBS- or LF-treated CB mononuclear cells $(n=3-6)$. Medium alone was used as control. In all plots, the results of individual experiments and mean \pm SD are shown. $P$ values were calculated using 2 -sided Student's $t$ tests ( $\mathbf{D}$ and $\mathbf{E}$ ) or 1-way ANOVA followed by Tukey-Kramer multiple-comparisons test (A-C and $\mathbf{F}$ ). ${ }^{*} P<$ $0.05 ;{ }^{* *} P<0.01 ;{ }^{* *} P<0.001$.

patterns were observed in human myeloid cells from either PB or CB from infants (Figure 5D). In humans, myeloid cells in preterm infants had only slightly lower expression of ITLN1 and LRP2 than those in full-term infants. Adults and infants with NEC had much lower expression of LF receptors (Figure $5 \mathrm{E}$ ).

To assess the contribution of individual LF receptors to the regulation of MDSC function by LF, we silenced $I t \ln 1$ and $L r p 2$ in BM cells by using shRNA lentiviral vectors (Supplemental Figure 7A). Silencing of $I t \ln 1$ did not affect LF-inducible expansion of myeloid cells from newborn mice, whereas Lrp2 shRNA completely abrogated it (Figure 5F and Supplemental Figure 7B). Downregulation of $L r p 2$, but not It $\ln 1$, abrogated LF-induced suppressive activity of PMN-MDSCs (Figure 5G) and M-MDSCs (Figure 5H). Downregulation of Lrp2 cancelled LF-induced increases in S100A9 (Figure 5I) and PGE2 (Figure 5J) in PMN-MDSCs. Increased production of
NO was the main mechanism of LF-mediated M-MDSC activity. Silencing of $L r p 2$ abrogated this effect (Figure 5K). Thus, the loss of sensitivity to LF by myeloid cells in adults could result from the downregulation of LF receptor Lrp2.

As we described, myeloid cells from adults did not respond to LF (Supplemental Figure 4). We tested to determine whether upregulation of Lrp2 can sensitize these cells to the effect of LF. To address this question, we overexpressed Lrp2 in adult mouse myeloid cells by using synergistic activation mediator CRISPRaCas9 (lentiSAMv2) lentivirus and different gRNA primers. After selection of 1 sgRNA that provided the best overexpression (Supplemental Figure 8A), we tested the effect of LF on these adult myeloid cells. We found that overexpression of Lrp2 sensitized adult BM cells to the effects of LF. It manifested in increased cell numbers (Supplemental Figure 8B) and acquisition of 
A

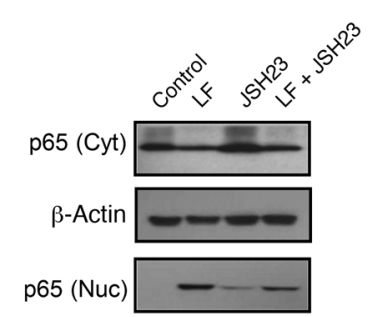

$\mathrm{a}-\mathrm{H} 3$ (Nuc)
B
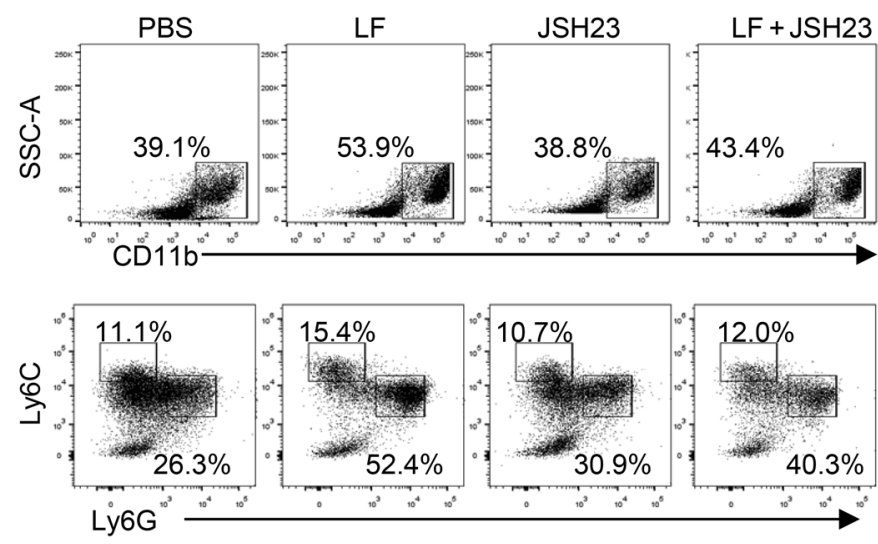
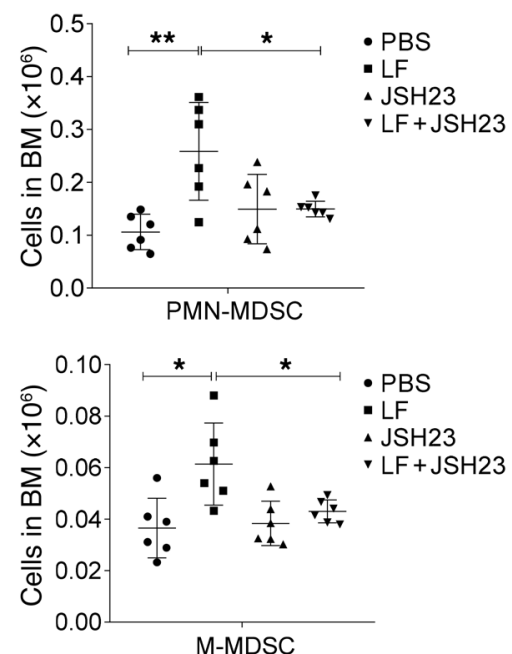

C

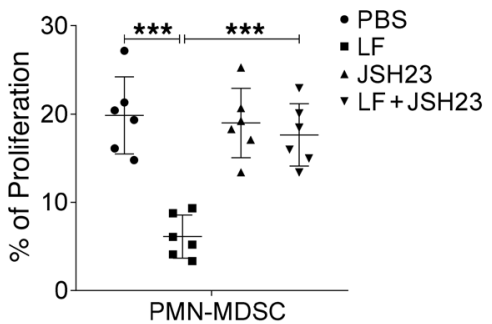

$\mathbf{F}$

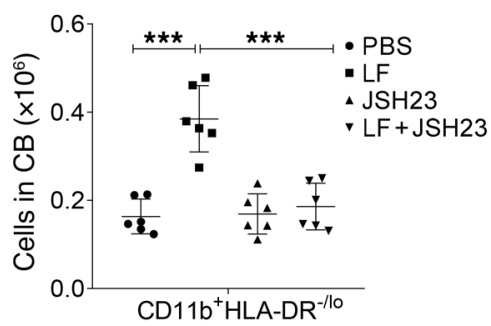

D

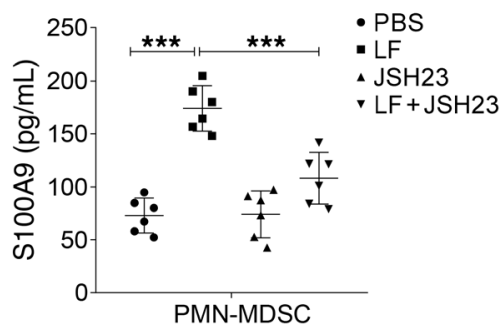

G

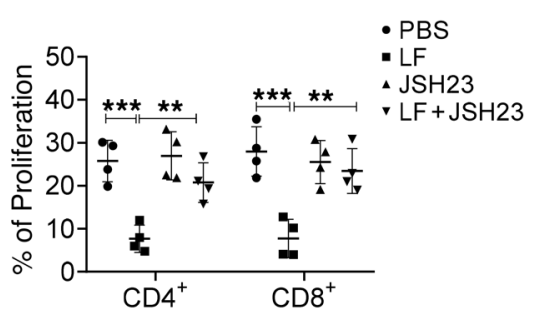

Figure 4. LF regulates MDSC function via NF-кB transcription factor. (A) Western blot of p65 in cytoplasm (Cyt) and nuclei (Nuc) in MDSCs from newborn mouse BM cells treated with LF and/or NF- $\mathrm{KB}$ inhibitor (JSH23, $2.5 \mu \mathrm{M})$. Control cells were treated with PBS. Typical example of 3 performed experiments is shown. (B) Typical example of staining (on the left) and absolute numbers of PMN-MDSCs and M-MDSCs (right) from mouse BM cells treated with LF and/ or JSH23 $(n=6)$. (C) Suppressive activity of PMN-MDSCs $(n=6)$. T cell proliferation of OT-I CD8 ${ }^{+}$T cells stimulated with SIINFEKL was measured in triplicate by CFSE staining. Mean \pm SD are shown. (D) The amount of S100A9 in PMN-MDSCs measured by ELISA. (E) The amount of PCE2 in PMN-MDSCs measured by ELISA $(n=6)$. (F) Absolute numbers of myeloid cells from LF-treated CB mononuclear cells in the presence or absence of JSH23 ( $n=6$ ). (C) Suppressive activity of myeloid cells from LF-treated CB cells in the presence or absence of JSH23. Effectors were CD4+ or CD8 ${ }^{+}$T cells stimulated with CD3/CD28 antibodies. Proliferation was measured in triplicate by CFSE staining. Results of individual experiments are shown $(n=4)$. In all plots, data represent mean \pm SD. $P$ values were calculated using 1-way ANOVA followed by Tukey-Kramer multiple-comparisons test. ${ }^{*} P<0.05 ;{ }^{* *} P<0.01 ;{ }^{* * *} P<0.001$.

immune-suppressive activity (Supplemental Figure 8, C and D). Previously, PPAR $\alpha$ and PPAR $\gamma$ were implicated in the upregulation of Lrp2 expression (32). We tested the effect of PPAR $\gamma$ agonist rosiglitazone on the expression of Lrp2 in myeloid cells and their sensitivity to LF. It was found that rosiglitazone substantially increased the expression of Lrp2 on PMNs (Supplemental Figure $8 \mathrm{E}$ ) and that treatment with LF converted those PMNs to suppressive PMN-MDSCs (Supplemental Figure 8F).

LF-MDSCs as the treatment of immune-mediated pathologies. Since a previous study implicated MDSCs in the control of NEC (21) and low levels of MDSCs correlated with the development of NEC in preterm infants (Figure 1B), we tested the possibility of using LF-MDSCs in the treatment of experimental NEC in mice.
NEC was induced in 1- to 3-day-old mice. BM cells from 2-weekold mice (lacking immune-suppressive activity) were treated for 48 hours with LF or vehicle alone in the presence of GM-CSF. The total population of $\mathrm{Gr}^{-1} \mathrm{C}^{+} \mathrm{CD} 11 \mathrm{~b}^{+}$MDSCs was isolated and injected i.p. into mice with NEC on 2 occasions during a 2-day interval (Supplemental Figure 9A). Administration of vehicle- or LF-treated myeloid cells resulted in equal accumulation of donor Gr- $1^{+} \mathrm{CD} 11 \mathrm{~b}^{+}$myeloid cells in the lamina propria of small intestine (Figure 6A). Intestinal inflammation was reduced in mice injected with vehicle-treated myeloid cells. However, this effect was substantially more pronounced after administration of LF-MDSCs (Figure 6B). Similar results were observed when intestinal permeability was assessed (Figure 6C). Injection of LF-MDSCs 
A

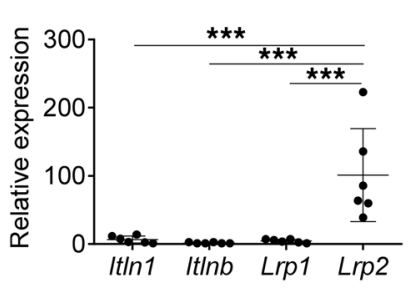

B

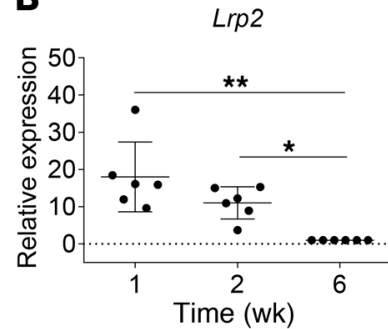

C

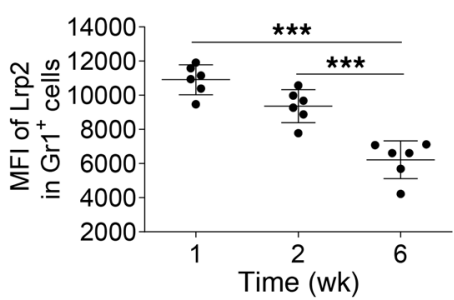

F PMN-MDSC
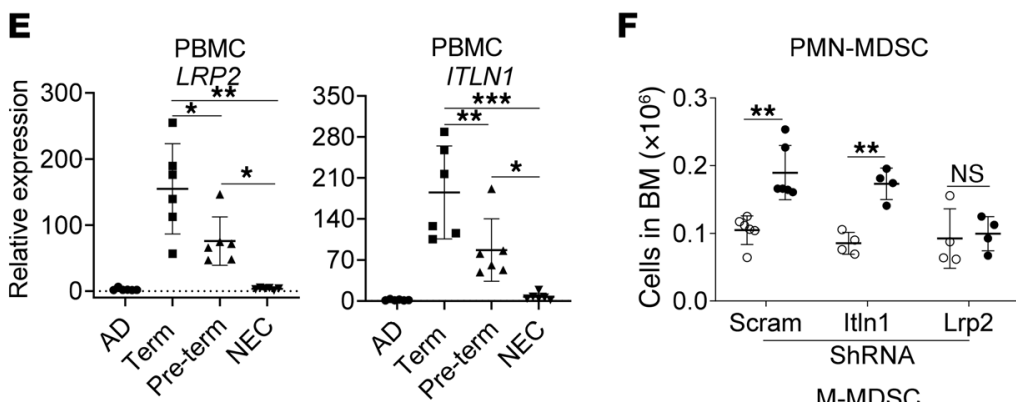

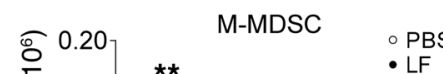

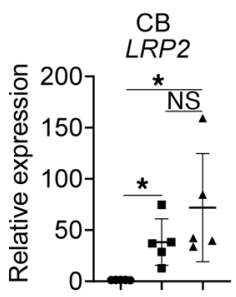

$\mathrm{CB}$
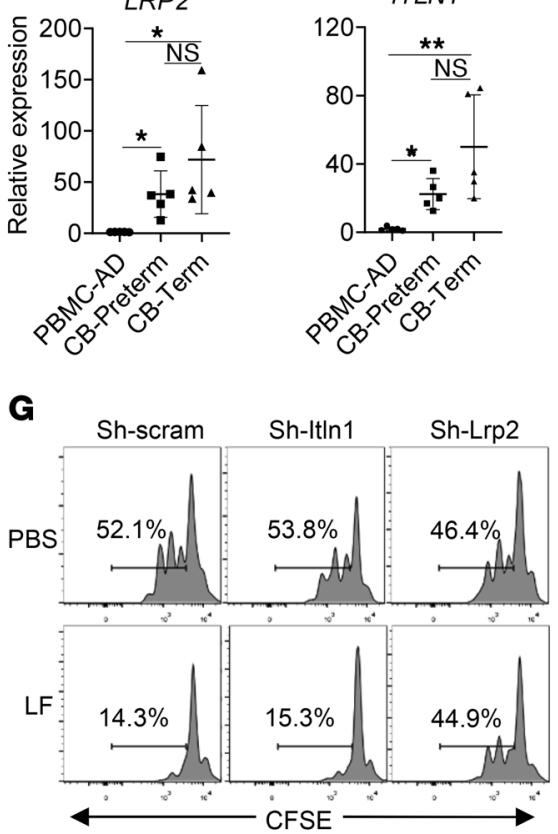

PMN-MDSC

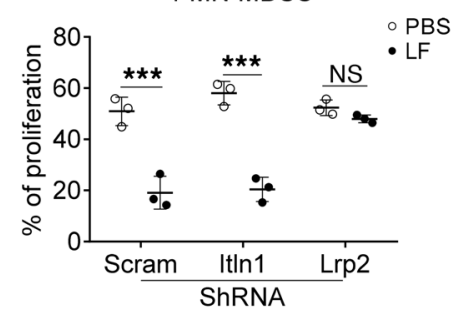

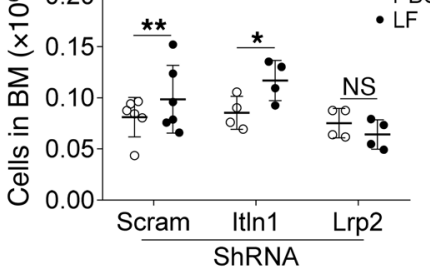

H
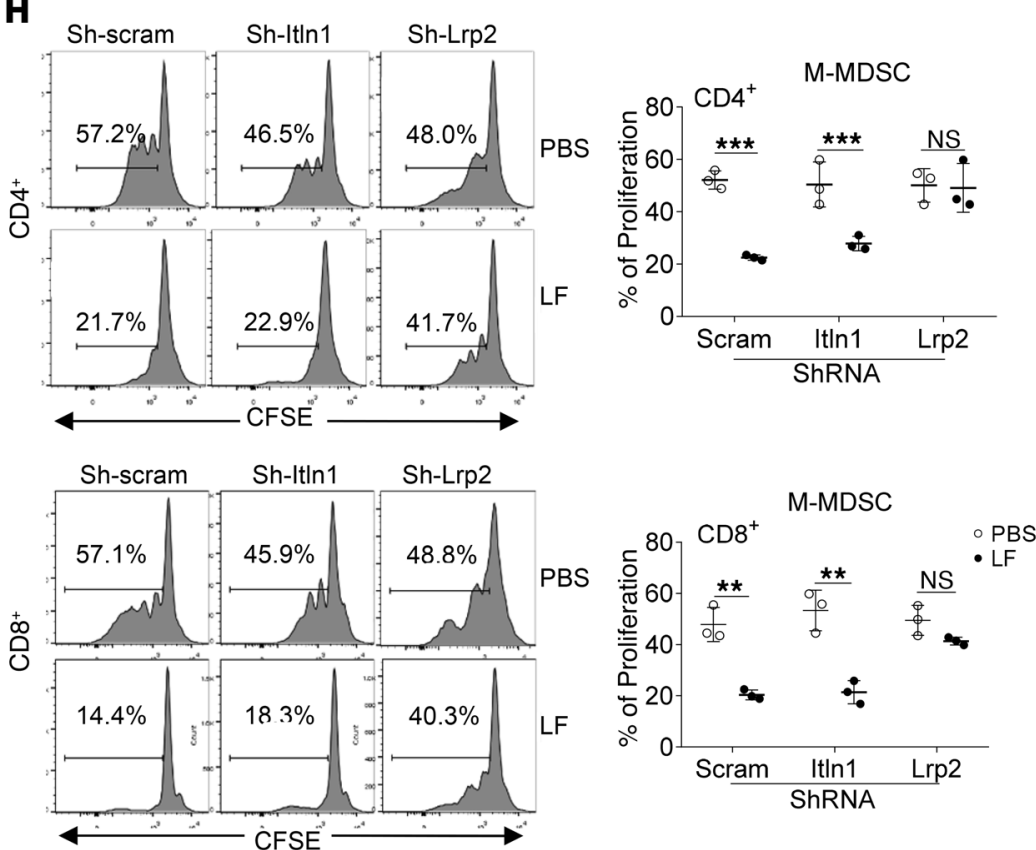

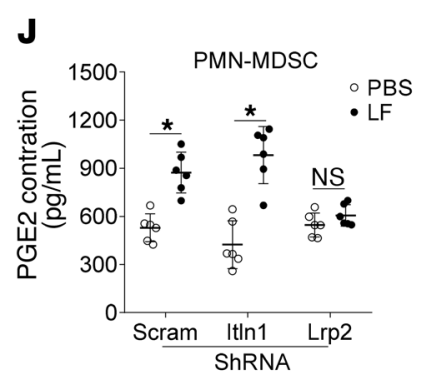

$\mathbf{K}$

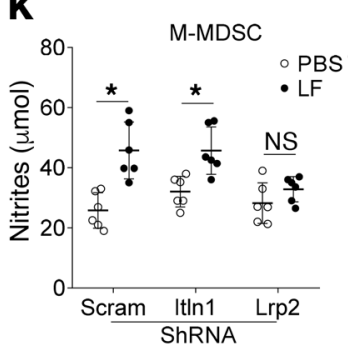


Figure 5. The effect of LF on myeloid cells is mediated by the LRP2 receptor. (A) Expression of indicated LF receptors in MDSCs from 2-weekold mice measured by qRT-PCR $(n=6)$. (B) Expression of $L r p 2$ in myeloid cells from mice of different ages measured by qRT-PCR $(n=6)$. (C) Surface expression of Lrp2 on mouse myeloid cells measured by flow cytometry $(n=6)$. (D) Expression of LF receptors in myeloid cells from PB (PBMC) or CB in full-term infants measured by qRT-PCR $(n=5)$. (E) Expression of ITLN1 and LRP2 in myeloid cells from PB of adults (AD) and full-term, preterm, and NEC infants or CB measured by qRT-PCR $(n=5-6)$. (F-K) Silencing of $I t \ln 1$ and $L r p 2$ in newborn mouse BM cells with shRNA or scramble shRNA (control) lentiviruses, followed by treatment with PBS or LF for 2 days. (F) The absolute numbers of PMN-MDSCs and M-MDSCs measured by flow cytometry $(n=4-6)$. (C) Suppressive activity of PMN-MDSCs. Effectors were OT-I CD8 ${ }^{+}$T cells stimulated with SIINFEKL. T cell proliferation was measured in triplicate by CFSE staining $(n=3)$. Upper panel shows typical example. Lower panel shows cumulative results. (H) Suppressive activity of M-MDSCs. Effectors were CD4+ or CD8 T cells stimulated with $\mathrm{CD} 3 / \mathrm{CD} 28$ antibodies $(n=3)$. Left panel shows typical example. Right panel shows cumulative results. (I) The amount of S100A9 in PMN-MDSCs measured by ELISA. (J) The amount of PGE2 in PMN-MDSCs measured by ELISA. (K) The amounts of nitrites in M-MDSCs measured by the Nitrite Assay Kit. $(\mathbf{I}-\mathbf{K}, n=6)$ Data represent individual results and mean \pm SD. $P$ values were calculated using 2 -sided Student' $s$ $t$ tests (F-K) or 1-way ANOVA followed by Tukey-Kramer multiple-comparisons test $(\mathbf{A}-\mathbf{E}) .{ }^{*} P<0.05$; ${ }^{* *} P<0.01$; ${ }^{* * *} P<0.001$.

into mice with NEC significantly $(P<0.0001)$ prolonged survival, whereas injection of vehicle-treated myeloid cells did not provide a therapeutic benefit $(P=0.07)$ and was markedly $(P=0.03)$ less effective than injection of LF-MDSCs (Figure 6D). Injection of LF-MDSCs significantly reduced the bacterial load in the intestine and PB of mice with NEC; this effect was stronger than the effect observed with control myeloid cells (Figure 6E). If LF had such a strong effect on myeloid cells, then administration of LF alone without myeloid cells may be sufficient to cause a therapeutic effect in mice with NEC. To test this hypothesis, we compared, side by side, the effect of administering LF-MDSCs (twice within a 3-day interval) to daily injections of $\mathrm{LF}$ (at a maximum tolerated dose of 0.25 $\mathrm{g} / \mathrm{kg})$. Treatment with LF-MDSCs caused significantly $(P<0.05)$ stronger inhibition of inflammation (Figure 6F), intestinal permeability (Figure 6G), and improvement of mouse survival (Figure $6 \mathrm{H})$ than injections of LF. Treatment of mice with LF-MDSCs was more efficient in reducing bacterial load in the intestine and blood of mice than treatment with LF alone (Figure 6I). Thus, LF-MDSCs were more effective for the treatment of NEC than LF alone.

Next, we tested the effect of the treatment with LF-MDSCs of different inflammatory conditions not limited to newborns. In these experiments, MDSCs were generated from 2-week-old mice with ex vivo treatment with LF as described above. Recipients were adult 6- to 8-week-old mice. In the model of OVA-induced lung inflammation (Supplemental Figure 9B), administration of LF-MDSCs caused a dramatic reduction in lung inflammation (Figure 7A) and decrease of serum IgE (Supplemental Figure 10A) as well as the cellular content of the bronchoalveolar lavage fluid (BALF) (Supplemental Figure 10B), proportion of eosinophils in BALF (Figure 7B) and concentration of IL-13 and IL- 4 in BALF (Supplemental Figure 10C). In the model of dextran sulfate sodiuminduced (DSS-induced) colitis (Supplemental Figure 9C), treatment with LF-MDSCs caused a dramatic reduction in inflammation (Figure 7C) and abrogated DSS-induced disease activity index (DAI) (Figure 7D), weight loss (Figure 7E), and decrease in length of colon (Supplemental Figure 10D). In the model of concanavalin A-induced (ConA-induced) hepatitis (Supplemental Figure 9D), LF-MDSCs reduced histological (Figure 7F) and enzymatic (alanine aminotransferase [ALT] and aspartate aminotransferase [AST]) signs of hepatitis (Figure 7G). Administration of LFMDSCs substantially reduced the presence of IFN- $\gamma$-producing $\mathrm{CD}^{+} \mathrm{T}$ cells (Supplemental Figure 10E) and the expression of Ifng, Tnfa, and Il6 (Supplemental Figure 10F) in liver tissues. Thus, LF-treated MDSCs provided strong therapeutic benefits in the array of inflammation-mediated diseases.

\section{Discussion}

This study presents evidence that MDSCs in newborns can be leveraged to control clinically relevant conditions marked by severe inflammation and associated tissue injury. MDSCs are pathologically activated PMNs and MONs with potent immune-suppressive activity that are implicated in the regulation of immune responses in cancer, chronic infections and inflammation, autoimmune diseases, etc. (19). Recent reports challenged the prevailing view that MDSCs are not present in steady-state conditions. We and others have demonstrated the existence of potently immunesuppressive MDSCs in healthy mouse and human neonates (2024). We hypothesized that MDSCs were evolutionarily developed to control potent inflammation associated with seeding of the gut microbiota during the first week of life. However, the clinical significance of MDSCs was uncertain. In this study, we found that the presence of PMN-MDSCs in the blood of preterm infants was substantially lower than that observed in full-term infants. Moreover, there was a strong negative correlation between the birth weight of the infants and the number of PMN-MDSCs, supporting the conclusion that the presence of MDSCs in newborns is inversely associated with their degree of immaturity. Importantly, the low number of PMN-MDSCs in preterm infants during the first 3 days of life was associated with the development of NEC at a later time. This suggests that MDSCs in newborns may have a clinical and potentially therapeutic significance. Late-onset infection $(>72$ hours after birth), NEC, and bronchopulmonary dysplasia are 3 of the most common and consequential sequalae of very preterm birth (<32 weeks' gestation) (33). Pathological inflammation is one of the major features of each of these morbidities and plays a critical role in determining the long-term outcomes associated with these conditions. Therefore, control of inflammation, together with the treatment of infection, could provide clinical benefit for these high-risk infants.

Exclusive breast milk feeding has been shown to reduce the risk of developing NEC in very preterm infants. Recently, accumulation of PMN-MDSCs with suppressive activity was found in breast milk (34). This finding is consistent with the role of PMN-MDSCs in the control of NEC described in our study. Our previous work indicated that the appearance of MDSCs in newborns was transitory during the first 10-12 days of life and was caused by LF (21). Consistent with those findings, we observed a direct correlation between the amount of LF in serum and the frequency of PMN-MDSCs in neonates. LF, a member of the transferrin family of iron-binding glycoproteins, is a cell-secreted mediator that has potent immune-regulatory activity and is a key 
A

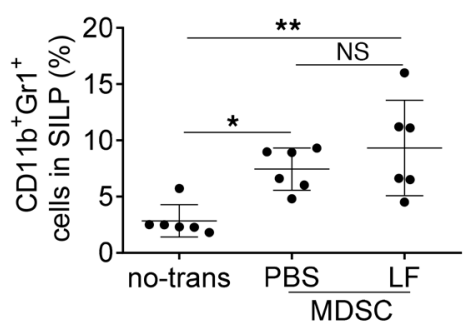

B

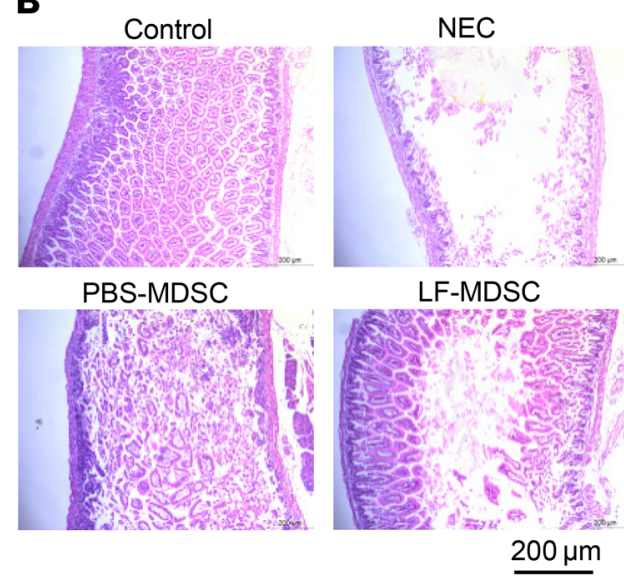

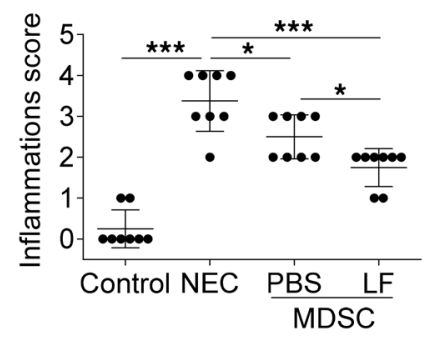

C
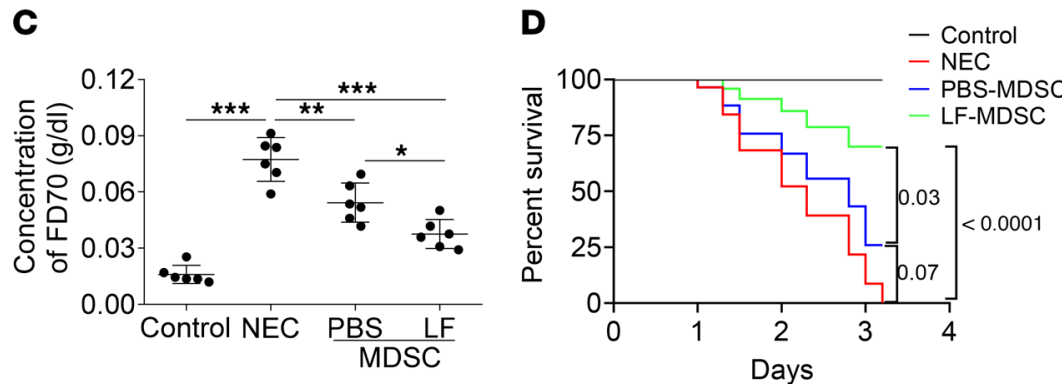

G
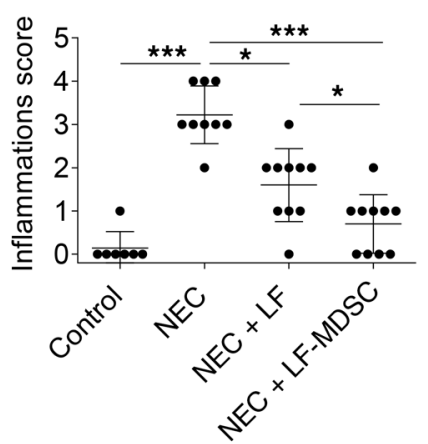

H

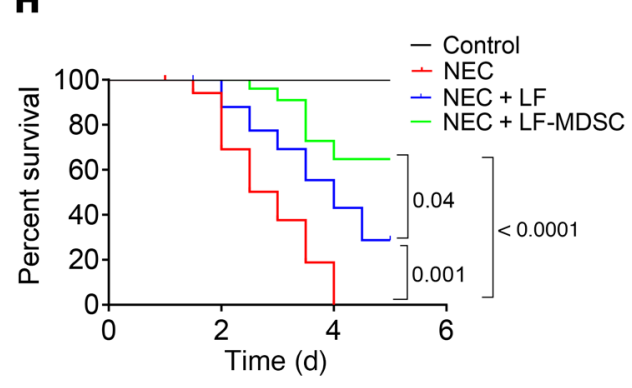

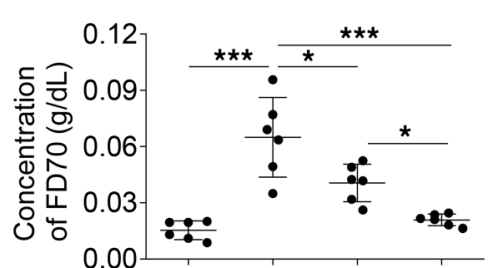

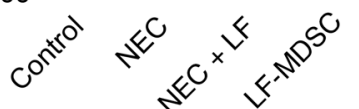
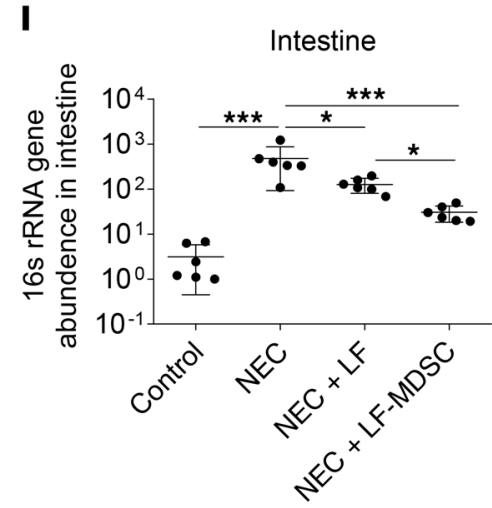

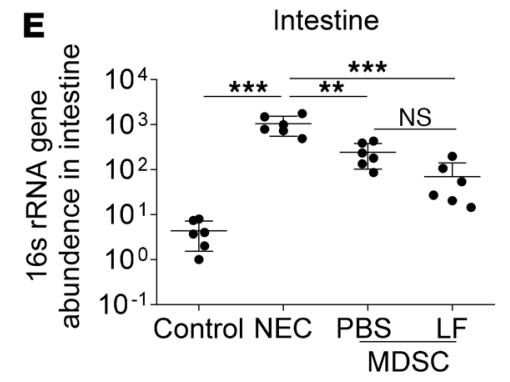

Blood

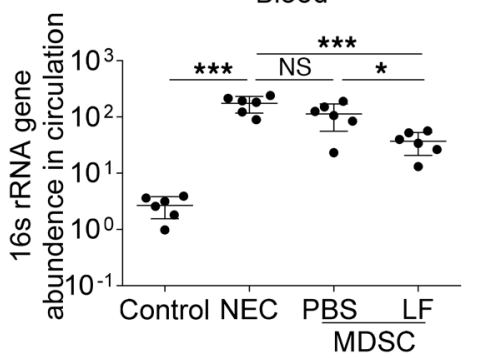

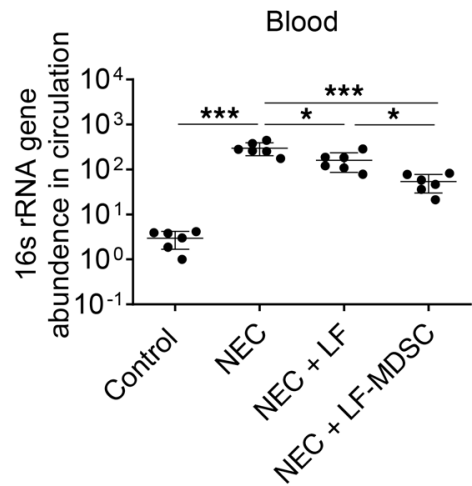


Figure 6. Therapeutic effect of LF-MDSCs in the model of NEC. (A-E) NEC was induced in 1- to 3-day-old mice as described in Supplemental Figure 9A. (A) Proportion of CFSE-labeled donor CD11 $\mathrm{b}^{+} \mathrm{Gr} 1^{+}$cells in small intestine lamina propria (SILP) of recipients $(n=6)$. (B) Representative H\&E staining of mouse intestine after NEC induction with or without transfer of MDSCs (left). Inflammation scores were calculated based on the severity of NEC (right) $(n=8)$. (C) FITC-labeled dextran (FD70) (reflecting intestine permeability) after NEC induction with or without transfer of MDSCs $(n=6)$. (D) Survival of mice after NEC induction with or without transfer of MDSCs $(n=28$ in each group). (E) Bacterial load in small intestine and blood after NEC induction with or without transfer of cells from PBS- or LF-treated BM cells $(n=6)$. (F-I) NEC was induced in 7-day-old mice treated with LF protein or transfer with LF-MDSCs. (F) Inflammation scores in the small intestine after NEC was induced and treated with LF protein or transfer of LF-MDSCs. (C) Detection of FITC-labeled dextran (FD70) after NEC was induced and treated with LF protein or transfer of LF-MDSCs $(n=6)$. (H) Survival of mice after NEC was induced and treated with LF protein or transfer of LF-MDSCs. Control, $n=40$; NEC, $n=37$; LF, $n=40$; LF-MDSCs, $n=40$. (I) Bacterial load in small intestine (left) and blood (right) after NEC was induced and treated with LF protein or transfer or LF-MDSCs ( $n=$ 6). In all plots, data represent mean \pm SD. $P$ values were calculated using 1-way ANOVA followed by Tukey-Kramer multiple-comparisons test (A-C, E-G, and I). ${ }^{*} P<0.05 ;{ }^{*} P<0.01 ;{ }^{*}{ }^{*} P<0.001$. Survival statistics were calculated using log-rank (Mantel-Cox) test.

component of the mammalian innate response to infection (31, 35). Our study demonstrated that PMNs and MONs from human and mouse neonates could be converted by LF, within 48 hours, to MDSCs with potent immune-suppressive activity. Importantly, this effect was observed not only in full-term infants, but also in preterm neonates. LF induced potent antibacterial activity in MDSCs, which could be exploited in future therapeutic applications. Activation of NF- $\mathrm{kB}$ transcription factor is a known downstream target of LF $(36,37)$. We confirmed upregulation of NF- $\mathrm{kB}$ in neonate myeloid cells by LF and demonstrated that selective inhibition of NF- $\mathrm{KB}$ abrogated the effect of LF.

No effect of LF was observed in cells obtained from adult volunteers and 6- to 8-week-old adult mice. We investigated the possibility that differences in the effect of LF on myeloid cells in newborns and adults could be the result of changes in the expression of LF receptors. Several LF-binding receptors have been previously described. Low-density LRP1 and LRP2 are considered primary LF receptors (38). The receptors for LF expressed on activated human lymphocytes (39) can be internalized by human MONs (40) and murine peritoneal macrophages (41), but the nature of the receptors on these cells was not clearly defined (42). The mechanism of LF endocytosis appears to be clathrin mediated (43). Human LF is capable of interacting with soluble CD14 to prevent CD14-LPS interaction (42). We found that newborn mice express only 1 common receptor for LF, Lrp2, and that its expression gradually decreased with age. Similar changes were observed in human neonates. In addition to LRP2, however, another receptor normally expressed in intestinal epithelia, ITLN1 (44), was also expressed. Downregulation of these receptors using shRNA demonstrated that Lrp2 was directly responsible for the effect of LF on the generation of MDSCs. Importantly, although expression of LRP2 in preterm infants was lower than in full-term neonates, it was still substantially higher than in adults. It was consistent with the fact that LF had a potent effect on the generation of MDSCs in preterm neonate myeloid cells. Upregu- lation of Lrp2 either by genetic manipulation or by the treatment of cells with PPAR $\gamma$ agonist sensitized adult myeloid cells to the conversion to MDSCs by LF.

These results suggested that LF can be used for the generation of MDSCs in newborns. These MDSCs not only inhibit T cells and blunt inflammation, but also have potent antibacterial activity. We tested the possibility of therapeutic utility of LF-MDSCs in an experimental model of NEC. BM PMNs and MONs from 2-weekold mice were used. At this age, mice lack functional MDSCs. We found that treatment of mice with LF-MDSCs had potent antiinflammatory activity and substantially prolonged the life of the mice. Importantly, LF-MDSCs markedly decreased bacterial load in the intestine and blood of mice with NEC. Moreover, LFMDSCs demonstrated potent antiinflammatory properties in other experimental models of inflammation more commonly observed outside the newborn period: autoimmune lung inflammation, colitis, and hepatitis. Considering that myeloid cells from adults can be made sensitive to LF after in vitro manipulation, this may open a possibility of therapeutic utility of LF-MDSCs, not only in neonates, but also in adults.

LF is present in human breast milk at a concentration of about $1 \mathrm{mg} / \mathrm{mL}$. Very preterm infants typically ingest little or no milk during the first few days after birth, resulting in low intake of LF (45). Therefore, enteral supplementation with bovine LF has been proposed as a simple strategy to compensate for this deficiency. However, despite demonstration in several small trials that supplementing the enteral diet of very preterm infants with LF prevents infection and associated complications, a recent large, randomized, placebo-controlled trial failed to establish clinical benefit of LF supplementation in this population (46). In our study, LF-MDSCs had much more potent antiinflammatory and antbacterial activity than LF alone, when used to treat experimental NEC. In late-onset NEC (induction in 7-day-old mice), LF-MDSCs substantially prolonged the life of the mice, whereas LF injections had a much smaller effect. One of the possible explanations of this phenomenon is the observation that LF could convert myeloid cells to MDSCs at only a relatively high dose $(>500$ $\mu \mathrm{g} / \mathrm{ml})$. This dose is much higher than that present in the serum of even full-term infants $(<30 \mu \mathrm{g} / \mathrm{ml})$ and may not be achievable through enteral supplementation. Thus, our study revealed the clinical significance of MDSCs in preterm infants and demonstrated that ex vivo LF-MDSCs have potent antiinflammatory and antibacterial activity that may be a powerful therapeutic tool in the treatment of inflammatory-mediated morbidity associated with very low-weight infants.

\section{Methods}

Human subjects. Samples of PB were collected from healthy adults, full-term infants, preterm infants, and patients with NEC (1 to 24 days old). Samples of CB were collected from full-term infants and preterm infants. In China, samples were collected from 111 subjects from the First Affiliated Hospital of SYSU, Guangzhou Women and Children's Medical Center, and the Third Affiliated Hospital of Guangzhou Medical University. In the US, samples were collected from 32 subjects ( 19 males and 13 females) at the CHOP and the Hospital of the University of Pennsylvania (HUP) (Supplemental Table 1). Preterm infants were defined as those with gestational age of less 
A
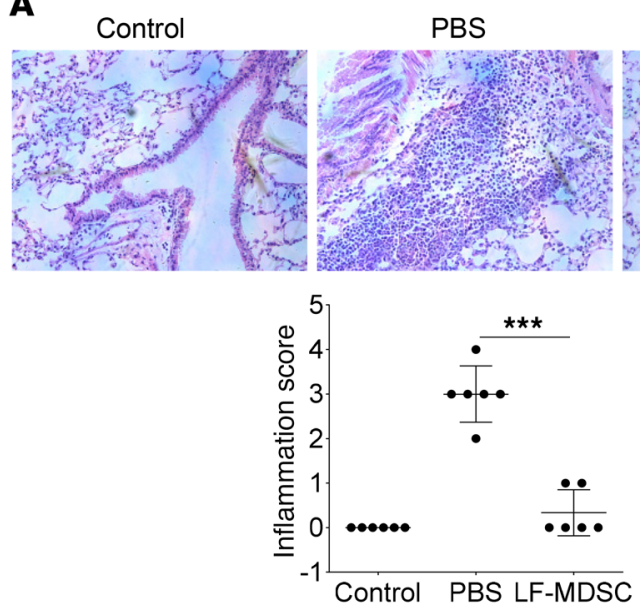

C
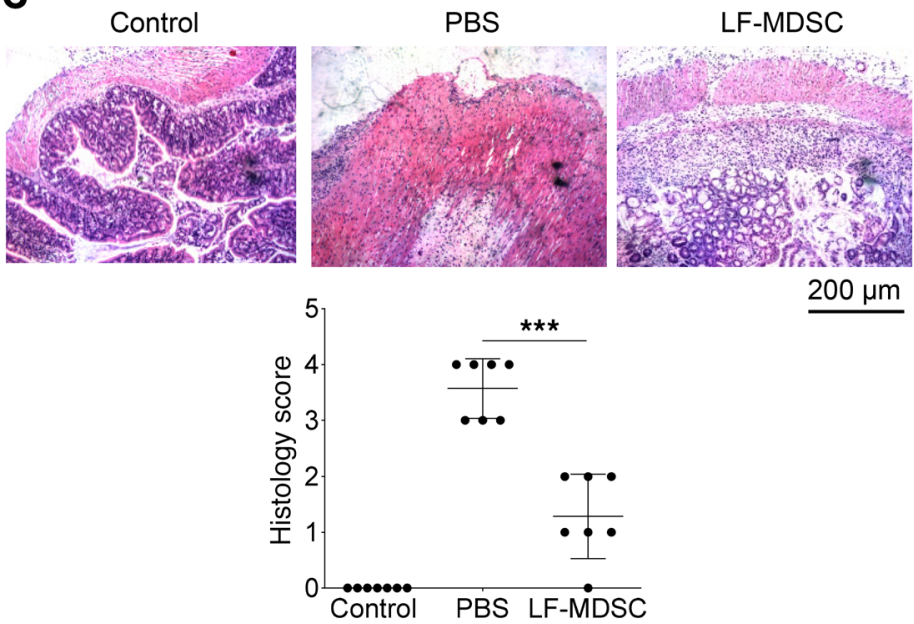

$\mathbf{F}$
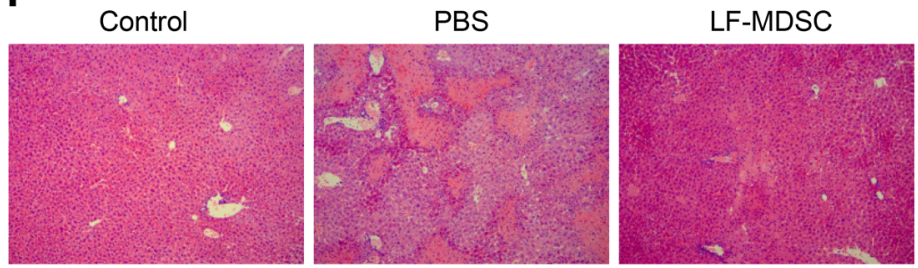

$200 \mu \mathrm{m}$

G
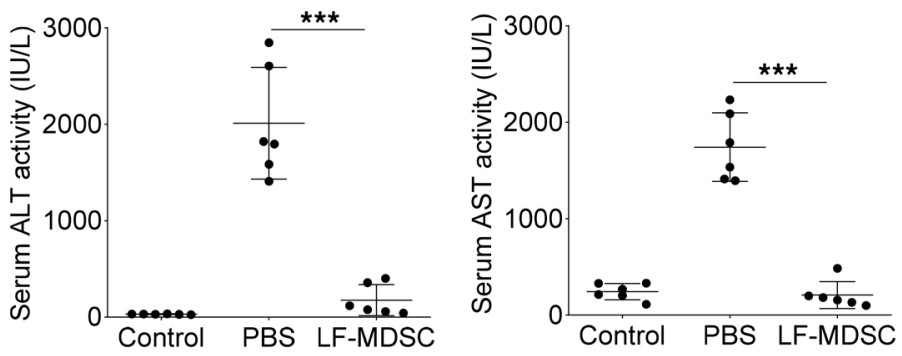

B

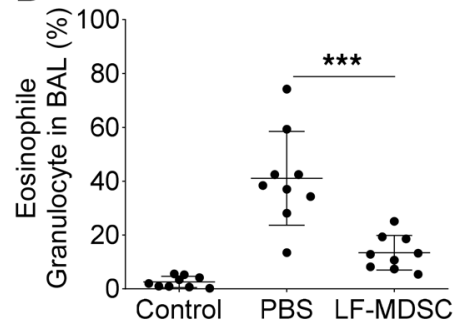

D

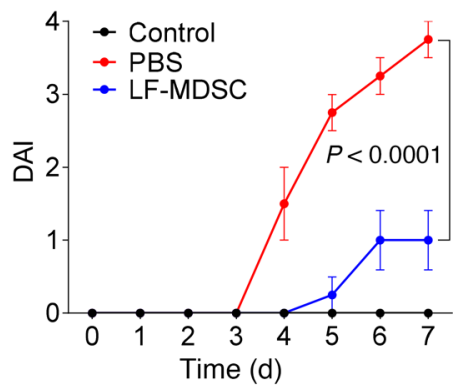

E

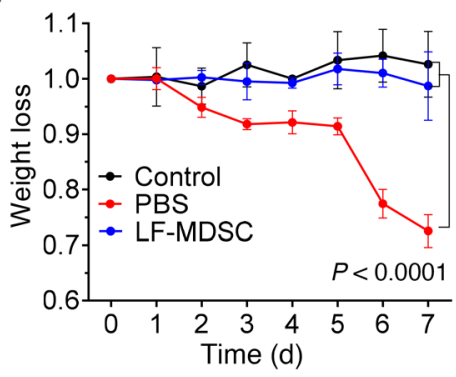

Figure 7. Therapeutic effect of LF-MDSCs under inflammatory conditions. ( $A$ and $\mathbf{B}$ ) Lung inflammation induced by OVA in 6- to 8-week-old adult mice. (A) Representative H\&E images of lungs (left) and inflammation scores (right) after lung inflammation induction with or without transfer of LF-MDSCs $(n=6)$. (B) Percentages of eosinophils in BALF $(n=9)$. (C-E) DSS-induced model of colitis in 6- to 8-week-old adult mice. (C) H\&E staining of colon (left) and histology scores (right) after DSS-induced colitis, with or without transfer of LF-MDSCs. $n=7$. (D and E) DAI (D) and loss of body weight (E) after colitis induction with or without transfer of exogenous LF-MDSCs were measured $(n=6)$. ( $\mathbf{F}$ and $\mathbf{G})$ Model of ConA-induced hepatitis in 6 - to 8-week-old adult mice. (F) H\&E staining of liver after induction of hepatitis with or without transfer of exogenous LF-MDSCs. (C) Levels of ALT (left) and AST (right) in serum $(n=6)$. In all plots, data represent individual results and mean \pm SD. $P$ values were calculated using 1-way ANOVA followed by Tukey-Kramer multiple-comparisons test (A, B, C, and $\mathbf{G}){ }^{* * *} P<0.001$. than 37 weeks. Full-term infants were defined as those with gestational age between 37 and 42 weeks.

Mice. C57BL/6 and BALB/c mice were obtained from the Laboratory Animal Center of SYSU, and OT-1 mice (C57BL/6-Tg(TCRaT-
CRb)1100mjb) were provided by Hui Zhang (SYSU). C57BL/6 mice were purchased from Taconic Biosciences and OT- 1 mice from the Jackson Laboratory. All mice were bred in pathogen-free facilities, and age-matched littermates were used as controls. 
Reagents. Reagents are listed in Supplemental Table 2.

Isolation of cells. Human mononuclear cells were isolated from $\mathrm{PB}$ or CB by Ficoll gradient. Mouse BM cells were isolated as described (47). Isolation of cells from intestine was performed by digestion in $0.5 \mathrm{mg} / \mathrm{ml}$ collagenase I and $5 \mathrm{U} / \mathrm{ml}$ DNase for 40 minutes after removal of intestinal epithelial cells. Leukocytes from lamina propria were enriched by a $40 \% / 80 \%$ Percoll gradient (48). For all samples, red blood cells were lysed using ACK buffer, and cell suspensions from all tissues were filtered through $70 \mu \mathrm{m}$ cell strainers before subsequent analysis.

Flow cytometric analysis and sorting. Single-cell suspensions were preincubated with anti-CD16/CD32 to block Fc receptors, spun down before cell-surface staining with specific antibodies. A cell-viability dye kit was used to exclude dead cells from subsequent analysis. Cells were incubated with antibodies (Supplemental Table 3) for 20 minutes at $4^{\circ} \mathrm{C}$ in the dark and then acquired on LSRFortessa or LSRII flow cytometers (BD Bioscience). For LRP2 staining, primary antibody (1:200 dilution) incubation was followed by a second antibody (goat anti-rabbit Alexa Fluor 647 conjugated at 1:200) incubation for 20 minutes at $4^{\circ} \mathrm{C}$ in dark. The data were analyzed by FlowJo V10 (Tree Star). For cell sorting, a BD FACSAria III cell sorter (BD Bioscience) was used. The gating strategy for mouse MDSCs was: CD11 $\mathrm{b}^{+} \mathrm{Ly} 6 \mathrm{C}^{\text {hi }}$ Ly $6 \mathrm{G}^{-}$for M-MDSCs and CD11b ${ }^{+}$Ly $6 \mathrm{C}^{-} \mathrm{Ly} 6 \mathrm{G}^{\text {hi }}$ for PMN-MDSCs.

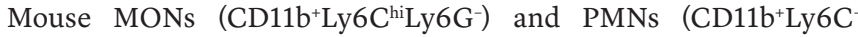
Ly $6 \mathrm{G}^{\text {hi) }}$ were isolated by sorting after $\mathrm{LF}$ treatment. For isolation of MONs and PMNs from human infants, mononuclear cell suspension from the PB was prepared by Ficoll gradient centrifugation and flow sorting was performed using FACSAria III based on the following markers: PMNs, CD $11 \mathrm{~b}^{+} \mathrm{HLA}-\mathrm{DR}^{-/ 10} \mathrm{CD} 15^{+} \mathrm{CD} 14^{-}$; MONs: $\mathrm{CD} 11 \mathrm{~b}^{+}$ HLA-DR ${ }^{-/ 10} \mathrm{CD} 14^{+} \mathrm{CD} 15^{-}$.

Immunosuppressive activity of MDSCs. Several methods were used. To evaluate M-MDSC-suppressive activity, sorted $\mathrm{CD}^{+} \mathrm{T}$ cells from spleen were labeled with CFSE $(2 \mu \mathrm{M})$ (Invitrogen), stimulated with anti-CD3-coated $(5 \mu \mathrm{g} / \mathrm{mL})$ plates and soluble anti-CD28 $(1 \mu \mathrm{g} /$ $\mathrm{mL}$ ) antibody (eBioscience), and cultured alone or with M-MDSCs at different ratios for 3 days. Cells were then stained with CD4-PECy5 and CD8a-PE antibodies, and $\mathrm{T}$ cell proliferation was analyzed by flow cytometry. T cell proliferation was also measured by ${ }^{3} \mathrm{H}$-thymidine uptake. For PMN-MDSC functional assay, $\mathrm{CD}^{+} \mathrm{T}$ cells were isolated from spleens of OT-I mice labeled with CFSE and then mixed with splenocytes from WT mice at a 1:4 ratio and incubated with PMN-MDSCs in the presence of cognate peptides (SIINFEKL).

For human samples, myeloid cells from $\mathrm{PB}$ or $\mathrm{CB}$ were plated on U-bottomed 96-well plates, cocultured with autologous $\mathrm{CD}^{+} \mathrm{T}$ cells, which were labeled with CFSE $(2 \mu \mathrm{M})$, and stimulated with CD3/CD28 antibodies. After 72 hours, cells were stained with anti-CD4-PE and antiCD8a-APC, and T cell proliferation was measured by flow cytometry.

LF treatment. For in vitro treatment, mouse BM cells were cultured in the presence of murine recombinant GM-CSF ( $20 \mathrm{ng} / \mathrm{ml})$ in RPMI 1640 medium (supplemented with 10\% FBS, $5 \mathrm{nM}$ glutamine, $25 \mathrm{mM}$ HEPES, and $1 \%$ antibiotics $)$ in the presence of LF protein $(500 \mu \mathrm{g} / \mathrm{ml})$ or PBS (control) for 1-3 days. For human samples, mononuclear cells from $\mathrm{PB}$ or CB were cultured in human recombinant GM-CSF (20 ng/ $\mathrm{ml}$ ) with $\mathrm{LF}$ protein $(500 \mu \mathrm{g} / \mathrm{ml})$ or PBS (control) for 1-2 days. Rosiglitazone was added at a final concentration of $2 \mu \mathrm{M}$ at the same time as for ON incubation; then PMN were extensively washed and used in antigen-specific suppression assays, as described above. For some

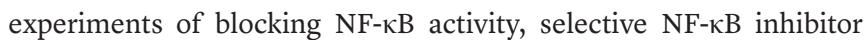
JSH23 was added. For in vivo treatment in mice, 1-week-old WT mice were injected i.p. with LF protein $(0.25 \mathrm{~g} / \mathrm{kg}$, in PBS) daily for 3 days.

Mouse NEC model. The NEC model was performed as previously described (21). One- to three-day-old mice received adult mouse cecum-derived bacteria $\left(7 \times 10^{7} \mathrm{CFU} /\right.$ mouse $)$ via gavage. From day 1 , mice were stressed by a hypoxia-cold shock cycle twice per day for 3 days, and then mice were returned to their cages and observed for NEC symptoms (characterized by lethargy, cyanosis, abdominal distention, and bloody stools). When mice displayed NEC symptoms, they were euthanized and a complete autopsy was performed. Small intestines were examined grossly and immediately placed in buffered formalin for H\&E staining. Pathological changes in intestine were scored by a blinded pathologist on a scale of 0 to 4 ; injury grades of 2, 3, or 4 were defined as NEC, with injury grades 3 and 4 defined as severe NEC.

Intestinal mucosa permeability. FITC-labeled dextran (FD70, Sigma-Aldrich) was used to assess intestinal mucosal permeability, as described previously (49). Briefly, mice from each experimental group were gavaged with $750 \mathrm{mg} / \mathrm{kg}$ FD70 suspended in PBS. The levels of FD70 were measured by spectra-fluorimeter after 4 hours. Levels of FD70 were measured by spectra-fluorimeter after 4 hours, and were calculated based on standard curve.

Bacterial loading in NEC model. Blood and intestine (stripped of feces) were collected from NEC mice, and total DNA was extracted. The amount of bacterial 16sRNA within the blood and intestine was then quantified by quantitative PCR (qPCR) as described previously (50).

Antimicrobial activity and phagocytosis. $1 \times 10^{5}$ PBS- or LFtreated MDSCs were incubated with $10^{7} \mathrm{CFU}$ of E. coli, C. albicans, or C. sakazakii for 2 hours, and bacterial growth was assessed 24 hours later as described (21). pHrodo Green E. coli BioParticles (Thermo Fisher, P35366) were used for the detection of phagocytic ingestion, as described previously (51). For the assessment of phagocytosis of MDSCs in vitro, PBS- or LF-treated MDSCs were cultured with E. coli BioParticles at $100 \mu \mathrm{g} / 1 \times 10^{5}$ cells for 90 minutes at $37^{\circ} \mathrm{C}, 5 \% \mathrm{CO}_{2}$. The reaction was stopped by washing with PBS, cells were stained with surface antibodies against MDSCs, and BioParticle internalization was evaluated by flow cytometry.

MDSC transfer. For MDSC transfer in the NEC model, $10^{6} \mathrm{LF}-$

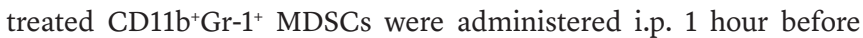
initiation of the NEC procedure at days 1 and 3; PBS-treated $\mathrm{CD} 11 \mathrm{~b}^{+} \mathrm{Gr}-1^{+}$cells were used as controls.

OVA-induced lung inflammation. Mice were sensitized i.p. with $5 \mu \mathrm{g} / \mathrm{g}$ OVA (grade V, Sigma-Aldrich) emulsified in $200 \mu \mathrm{g} / \mathrm{g}$ of aluminum hydroxide on days 0 and 7 , followed by intranasal instillation with OVA ( $5 \mu \mathrm{g} / \mu \mathrm{l}$ of PBS) on days 17 to 21 . Mice were sacrificed for analysis 24 hours after the last challenge. For MDSC transfer, $10^{6}$ LF-treated $\mathrm{CD} 11 \mathrm{~b}^{+} \mathrm{Gr}-1^{+}$MDSCs were injected into recipient mice i.v. at days -1 and 6 before each sensitization.

Induction of murine colitis by DSS. Mice were given $2.5 \%$ (w/v) DSS (MP Biomedicals) in drinking water for 7 days; this was then changed to regular drinking water. Mice were weighed every day to determine weight loss. The clinical scoring of a DAI that ranged from 0 to 4 was calculated as described previously (52). Colon length was measured and fixed in buffered paraformaldehyde for H\&E staining. Histological quantification was done in a blinded fashion. For MDSC transfer, $10^{6}$ enriched LF-induced CD11 $\mathrm{b}^{+} \mathrm{Gr}-1^{+}$MDSCs were injected into recipient mice i.v. at days 2 and 5 . 
ConA-induced murine hepatitis. We followed the procedures described earlier (53). Mice were given a single i.v. injection of ConA (Sigma-Aldrich) at a dose of $20 \mathrm{mg} / \mathrm{kg}$ body weight and were sacrificed 24 hours later. $10^{6}$ LF-MDSCs were injected i.v. into recipient mice before ConA injection. Liver tissues were fixed in buffered paraformaldehyde for H\&E staining. Hepatic mononuclear cells (HMCs) were isolated on Percoll gradient, and the cells were stained with specific antibodies, including anti-CD45-PE and anti-CD4-PE-cy7. For intracellular staining, the cells were fixed and permeabilized in Fix/Perm Buffer (BD Biosciences) and then stained with anti-IFN- $\gamma$-APC antibody. Serum was collected, and the levels of ALT and AST were measured by an automatic biochemical analyzer (Beckman Coulter, CX9).

Real-time qPCR. Total RNA was isolated using the RNeasy Mini Kit (QIAGEN, catalog 74104). $1 \mu \mathrm{g}$ of total RNA was reverse-transcribed using the PrimeScript RT Reagent Kit (Takara, catalog RR037Q) at $37^{\circ} \mathrm{C}$ for 15 minutes. qPCR was run on a Bio-Rad CFX96 PCR System (Bio-Rad) using SYBR Premix Ex TaqTM (Takara). The mRNA levels of specific genes were determined by the relative standard curve method, normalized against $\beta$-actin levels. qPCR analyses were done in triplicate, and experiments were repeated at least twice. Primers are listed in Supplemental Table 4.

ELISA. $2 \times 10^{6} / \mathrm{ml}$ cells were cultured for 48 hours in RPMI complete medium; the amounts of PGE2 and S100A9 in the cell lysates were measured by ELISA according to the manufacturer's protocol (Invitrogen and R\&D Systems, respectively). For human LF, plasma was collected and the concentration of LF was measure by ELISA.

NO production. The NO content in culture supernatants was measured with the Nitrite Assay Kit following the manufacturer's protocol (Griess reagent) (K544-200, BioVision) (54).

Western blotting. Cells were lysed with $10 \mu \mathrm{l}$ lysis buffer in the presence of protease and phosphatase inhibitors. Equal amounts of cellular proteins were subjected to $12 \%$ SDS-PAGE and transferred to PVDF membranes. After blocking, membranes were immunoblotted with the indicated primary Abs and secondary Abs. Results were visualized using ECL detection. All antibodies are listed in Supplemental Table 2.

shRNAs, lentivirus production, and infection of BM cells. shRNA sequences against Lrp2 and Itln1 were obtained from the SigmaAldrich Mission shRNA Library (sequences listed in Supplemental Table 4) and cloned into the lentiviral vector pLKO.3G. Then the 293T cells were cotransfected with shRNA vector and lentiviral packaging plasmids (psPAX2 and pMD2.G). At 48 and 72 hours, the supernatant containing virus was harvested, filtered through a $0.45 \mathrm{~mm}$ filter, and mixed with $5 \mu \mathrm{g} / \mathrm{ml}$ polybrene (Sigma-Aldrich, catalog H9268). For infection, BM cells were cultured overnight, and supernatants were aspirated and replaced with lentiviral supernatants, followed by centrifugation (900 $\mathrm{g}$ for 45 minutes). After 6 hours of infection, medium was changed. Infection was repeated the next day, and then cells were treated with PBS or LF for another 2 days.

Synergistic activation mediator CRISPRa-Cas9 (lentiSAMv2) lentivirus was used to overexpress the Lrp-2 gene in adult mouse BM cells. Six sgRNA-specific primers for the Lrp-2 gene (Supplemental Table 4) were obtained from the Synergistic Activation Mediator (SAM) sgRNA library (55) and cloned into the lentiviral vector lentiSAMv2. 293T cells were transfected with the lentiviral vector-expressing sgRNA (15 $\mu \mathrm{g})$, the packaging plasmid psPAX2 $(12.3 \mu \mathrm{g})$, and the envelope expression plasmid pMD2.G $(6.7 \mu \mathrm{g})$ to produce lentiviral particles. BM cells from adult mice were infected with lentiviral particles, followed by LF treatment for 48 hours before measurement of MDSC levels and their immunosuppressive function. Lentiviral vectors lacking any sgRNA were used as a no-guide control.

Statistics. Statistical analyses were performed using 2-tailed Student's $t$ tests for comparing 2 groups. One-way ANOVA followed by Tukey-Kramer multiple comparisons test was performed for comparing 3 or more groups within the same experiment. Survival curves were compared with the log-rank (Mantel-Cox) test and Gehan-Breslow-Wilcoxon test. Statistical tests were performed in GraphPad Prism, version 8.0, software (GraphPad Software Inc.). Correlations between different parameters were analyzed using Spearman's rank test. All data are presented as mean \pm SD, and a $P$ value of less than 0.05 was considered significant.

Study approval. The study was approved by the clinical ethics review boards of SYSU, Guangzhou Women and Children's Medical Center, the Third Affiliated Hospital of Guangzhou Medical University, and CHOP. All participants or legal guardians of the participants signed informed consent forms. Animal experiments were approved by the SYSU Animal Care and Use Committee. Experiments performed at Wistar Institute were approved by the IACUC of the Wistar Institute.

\section{Author contributions}

DIG and JZ conceived and supervised the study and wrote the manuscript. YL, MP, QX, SF, YH, JH, and XL performed experiments. WL, YT, XL, WY, WZ, FW, CJ, QC, and EAJ provided clinical resources, collected patients' information, and contributed to writing the manuscript. GSW contributed to writing the manuscript. Authors with equal contribution listed in alphabetical order.

\section{Acknowledgments}

This work was supported by the Wistar Institute Animal and Flow Cytometry Core Facilities (NIH grant P30 CA010815-50) as well as a CHOP pilot grant (to DIG). It was also supported by grants from the following institutions (to JZ): High-Level Talent Start-Up Funding of Tianjin Medical University, National Natural Science Foundation of China (grants 81571520, 81771665, and 91542112), and National Natural Science Foundation of Guangdong (2017B030311014).

Address correspondence to: Dmitry I. Gabrilovich, 3601 Spruce Street, Philadelphia, Pennsylvania 19104-4265, USA. Phone: 215.495.6955; Email: dgabrilovich@wistar.org. Or to: Jie Zhou, 22 Qixiangtai Road, Tianjin, China, 300070. Phone:86.22.8333.6137; Email:zhoujie@tmu.edu.cn.
1. Payne NR, Carpenter JH, Badger GJ, Horbar JD, Rogowski J. Marginal increase in cost and excess length of stay associated with nosocomial bloodstream infections in surviving very low birth weight infants. Pediatrics. 2004;114(2):348-355.
2. Ganapathy V, Hay JW, Kim JH. Costs of necrotizing enterocolitis and cost-effectiveness of exclusively human milk-based products in feeding extremely premature infants. Breastfeed Med. 2012;7(1):29-37.
3. Stey A, et al. Outcomes and costs of surgical treatments of necrotizing enterocolitis. Pediatrics. 2015;135(5):e1190-e1197.

4. Johnson TJ, Patel AL, Jegier BJ, Engstrom JL, Meier PP. Cost of morbidities in very low birth 
weight infants. J Pediatr. 2013;162(2):243-249.e1.

5. Niño DF, Sodhi CP, Hackam DJ. Necrotizing enterocolitis: new insights into pathogenesis and mechanisms. Nat Rev Gastroenterol Hepatol. 2016;13(10):590-600.

6. Neu J, Walker WA. Necrotizing enterocolitis. NEngl JMed. 2011;364(3):255-264.

7. Holman RC, Stoll BJ, Curns AT, Yorita KL, Steiner CA, Schonberger LB. Necrotising enterocolitis hospitalisations among neonates in the United States. Paediatr Perinat Epidemiol. 2006;20(6):498-506.

8. Guillet R, et al. Association of H2-blocker therapy and higher incidence of necrotizing enterocolitis in very low birth weight infants. Pediatrics. 2006;117(2):e137-e142.

9. Stoll BJ, et al. Trends in care practices, morbidity, and mortality of extremely preterm neonates, 1993-2012. JAMA. 2015;314(10):1039-1051.

10. Fitzgibbons SC, et al. Mortality of necrotizing enterocolitis expressed by birth weight categories. J Pediatr Surg. 2009;44(6):1072-1075; discussion 1075 .

11. Hintz SR, et al. Neurodevelopmental and growth outcomes of extremely low birth weight infants after necrotizing enterocolitis. Pediatrics. 2005;115(3):696-703.

12. Salhab WA, Perlman JM, Silver L, Sue Broyles R. Necrotizing enterocolitis and neurodevelopmental outcome in extremely low birth weight infants $<1000$ g. JPerinatol. 2004;24(9):534-540.

13. Stoll BJ, et al. Late-onset sepsis in very low birth weight neonates: the experience of the NICHD Neonatal Research Network. Pediatrics. 2002;110(2 Pt 1):285-291.

14. Zhang X, Zhivaki D, Lo-Man R. Unique aspects of the perinatal immune system. Nat Rev Immunol. 2017;17(8):495-507.

15. Gabrilovich DI, Ostrand-Rosenberg S, Bronte V. Coordinated regulation of myeloid cells by tumours. Nat Rev Immunol. 2012;12(4):253-268.

16. Umansky V, Blattner C, Gebhardt C, Utikal J. The role of myeloid-derived suppressor cells (MDSC) in cancer progression. Vaccines (Basel). 2016;4(4):E36.

17. Youn JI, Collazo M, Shalova IN, Biswas SK, Gabrilovich DI. Characterization of the nature of granulocytic myeloid-derived suppressor cells in tumor-bearing mice. J Leukoc Biol. 2012;91(1):167-181.

18. Zhang YL, et al. Peripheral blood MDSC, IL-10 and IL-12 in children with asthma and their importance in asthma development. PLOS ONE. 2013;8(5):e63775.

19. Veglia F, Perego M, Gabrilovich D. Myeloidderived suppressor cells coming of age. Nat Immunol. 2018;19(2):108-119.

20. Schwarz J, et al. Granulocytic myeloid-derived suppressor cells (GR-MDSC) accumulate in cord blood of preterm infants and remain elevated during the neonatal period. Clin Exp Immunol. 2018;191(3):328-337.

21. He YM, et al. Transitory presence of myeloidderived suppressor cells in neonates is critical for control of inflammation. Nat Med. 2018;24(2):224-231.

22. Gervassi A, et al. Myeloid derived suppressor cells are present at high frequency in neonates and suppress in vitro T cell responses. PLOS ONE. 2014;9(9):e107816.

23. Rieber N, et al. Neutrophilic myeloid-derived suppressor cells in cord blood modulate innate and adaptive immune responses. Clin Exp Immunol. 2013;174(1):45-52.

24. Köstlin N, et al. Granulocytic myeloid-derived suppressor cells from human cord blood modulate T-helper cell response towards an anti-inflammatory phenotype. Immunology. 2017;152(1):89-101.

25. Leiber A, et al. Neonatal myeloid derived suppressor cells show reduced apoptosis and immunosuppressive activity upon infection with Escherichia coli. Eur J Immunol. 2017;47(6):1009-1021.

26. Heinemann AS, et al. In neonates S100A8/S100A9 alarmins prevent the expansion of a specific inflammatory monocyte population promoting septic shock. FASEB J. 2017;31(3):1153-1164.

27. Ulas T, et al. S100-alarmin-induced innate immune programming protects newborn infants from sepsis. Nat Immunol. 2017;18(6):622-632.

28. Condamine T, et al. Lectin-type oxidized LDL receptor-1 distinguishes population of human polymorphonuclear myeloid-derived suppressor cells in cancer patients. Sci Immunol. 2016;1(2):aaf8943.

29. Bronte V, et al. Recommendations for myeloid-derived suppressor cell nomenclature and characterization standards. Nat Commun. 2016;7:12150.

30. Ando K, et al. Human lactoferrin activates NF-kappaB through the Toll-like receptor 4 pathway while it interferes with the lipopolysaccharide-stimulated TLR4 signaling. FEBS J. 2010;277(9):2051-2066.

31. Actor JK, Hwang SA, Kruzel ML. Lactoferrin as a natural immune modulator. Curr Pharm Des. 2009;15(17):1956-1973.

32. Cabezas F, Lagos J, Céspedes C, Vio CP, Bronfman M, Marzolo MP. Megalin/LRP2 expression is induced by peroxisome proliferator-activated receptor -alpha and -gamma: implications for PPARs' roles in renal function. PLOS ONE. 2011;6(2):e16794

33. Shane AL, Sánchez PJ, Stoll BJ. Neonatal sepsis. Lancet. 2017;390(10104):1770-1780.

34. Köstlin N, et al. Granulocytic myeloid-derived suppressor cells (GR-MDSC) in breast milk (BM); GR-MDSC accumulate in human BM and modulate T-cell and monocyte function. Front Immunol. 2018;9:1098.

35. Vogel HJ. Lactoferrin, a bird's eye view. Biochem Cell Biol. 2012;90(3):233-244.

36. Zemankova N, Chlebova K, Matiasovic J, Prodelalova J, Gebauer J, Faldyna M. Bovine lactoferrin free of lipopolysaccharide can induce a proinflammatory response of macrophages. BMC Vet Res. 2016;12(1):251.

37. Mantuano E, Brifault C, Lam MS, Azmoon P, Gilder AS, Gonias SL. LDL receptor-related protein-1 regulates NFKB and microRNA-155 in macrophages to control the inflammatory response. Proc Natl Acad Sci U S A. 2016;113(5):1369-1374.

38. Grey A, et al. The low-density lipoprotein receptor-related protein 1 is a mitogenic receptor for lactoferrin in osteoblastic cells. Mol Endocrinol. 2004;18(9):2268-2278.
39. Mazurier J, Legrand D, Hu WL, Montreuil J, Spik G. Expression of human lactotransferrin receptors in phytohemagglutinin-stimulated human peripheral blood lymphocytes. Isolation of the receptors by antiligand-affinity chromatography. Eur J Biochem. 1989;179(2):481-487.

40. Birgens HS, Hansen NE, Karle H, Kristensen LO. Receptor binding of lactoferrin by human monocytes. Br J Haematol. 1983;54(3):383-391.

41. van Snick JL, Markowetz B, Masson PL. The ingestion and digestion of human lactoferrin by mouse peritoneal macrophages and the transfer of its iron into ferritin. J Exp Med. 1977;146(3):817-827.

42. Suzuki YA, Lopez V, Lönnerdal B. Mammalian lactoferrin receptors: structure and function. Cell Mol Life Sci. 2005;62(22):2560-2575.

43. Lopez V, Kelleher SL, Lonnerdal B. Lactoferrin receptor mediates apo- but not holo-lactoferrin internalization via clathrin-mediated endocytosis in trophoblasts. Biochem J. 2008;411(2):271-278.

44. Akiyama Y, et al. A lactoferrin-receptor, intelectin 1 , affects uptake, sub-cellular localization and release of immunochemically detectable lactoferrin by intestinal epithelial Caco- 2 cells. J Biochem. 2013;154(5):437-448.

45. Cuenca AG, Wynn JL, Moldawer LL, Levy O. Role of innate immunity in neonatal infection. Am J Perinatol. 2013;30(2):105-112.

46. ELFIN trial investigators group. Enteral lactoferrin supplementation for very preterm infants: a randomised placebo-controlled trial. Lancet. 2019;393(10170):423-433.

47. Lei AH, et al. ICAM-1 controls development and function of ILC2. JExp Med. 2018;215(8):2157-2174.

48. Seehus CR, et al. The development of innate lymphoid cells requires TOX-dependent generation of a common innate lymphoid cell progenitor. Nat Immunol. 2015;16(6):599-608.

49. Rager TM, Olson JK, Zhou Y, Wang Y, Besner GE. Exosomes secreted from bone marrow-derived mesenchymal stem cells protect the intestines from experimental necrotizing enterocolitis. JPediatr Surg. 2016;51(6):942-947.

50. Kubinak JL, et al. MyD88 signaling in T cells directs IgA-mediated control of the microbiota to promote health. Cell Host Microbe. 2015;17(2):153-163.

51. Davis RJ, Silvin C, Allen CT. Avoiding phagocytosis-related artifact in myeloid derived suppressor cell T-lymphocyte suppression assays. J Immunol Methods. 2017;440:12-18.

52. Yang JY, et al. Enteric viruses ameliorate gut inflammation via Toll-like receptor 3 and Tolllike receptor 7-mediated interferon- $\beta$ production. Immunity. 2016;44(4):889-900.

53. Ju Y, et al. The Tim-3/galectin-9 pathway involves in the homeostasis of hepatic Tregs in a mouse model of concanavalin A-induced hepatitis. $\mathrm{Mol}$ Immunol. 2014;58(1):85-91.

54. Corzo CA, et al. HIF-1 $\alpha$ regulates function and differentiation of myeloid-derived suppressor cells in the tumor microenvironment. JExp Med. 2010;207(11):2439-2453.

55. Joung J, et al. Genome-scale CRISPR-Cas 9 knockout and transcriptional activation screening. Nat Protoc. 2017;12(4):828-863. 